\title{
Eta-diagonal distributions and infinite divisibility for R-diagonals
}

\author{
Hari Bercovici ${ }^{\mathrm{a}, 1}$, Alexandru Nica ${ }^{\mathrm{b}, 2}$, Michael Noyes ${ }^{\mathrm{c}}$ and Kamil Szpojankowski ${ }^{\mathrm{d}}$ \\ ${ }^{\mathrm{a}}$ Department of Mathematics, Indiana University, Bloomington, Indiana 47405, USA. E-mail: bercovic@indiana.edu \\ ${ }^{\mathrm{b}}$ Department of Pure Mathematics, University of Waterloo, Ontario N2L 3G1, Canada. E-mail: anica@ uwaterloo.ca \\ ${ }^{\mathrm{c}}$ Department of Mathematics, Bard High School Early College, New York 10002, New York, USA. E-mail: mnoyes@bhsec.bard.edu \\ ${ }^{\mathrm{d}}$ Department of Pure Mathematics, University of Waterloo, Ontario N2L 3G1, Canada \\ and Faculty of Mathematics and Information Science, Warsaw University of Technology, 00-661 Warszawa, Poland. \\ E-mail: kszpojan@uwaterloo.ca; k.szpojankowski@mini.pw.edu.pl
}

Received 29 August 2016; revised 13 February 2017; accepted 7 March 2017

\begin{abstract}
The class of $R$-diagonal $*$-distributions is fairly well understood in free probability. In this class, we consider the concept of infinite divisibility with respect to the operation $\boxplus$ of free additive convolution. We exploit the relation between free probability and the parallel (and simpler) world of Boolean probability. It is natural to introduce the concept of an $\eta$-diagonal distribution that is the Boolean counterpart of an $R$-diagonal distribution. We establish a number of properties of $\eta$-diagonal distributions, then we examine the canonical bijection relating $\eta$-diagonal distributions to infinitely divisible $R$-diagonal ones. The overall result is a parametrization of an arbitrary $\boxplus$-infinitely divisible $R$-diagonal distribution that can arise in a $C^{*}$-probability space by a pair of compactly supported Borel probability measures on $[0, \infty)$. Among the applications of this parametrization, we prove that the set of $\boxplus$-infinitely divisible $R$-diagonal distributions is closed under the operation $\otimes$ of free multiplicative convolution.
\end{abstract}

Résumé. Dans le cadre des probabilités libres, la classe des $*$-distributions $R$-diagonales est assez bien comprise. Dans cette classe, nous nous intéressons au concept d'infinie divisibilité par rapport à l'opération de convolution additive libre $\boxplus$. Nous exploitons la relation entre probabilités libres et le monde parallèle (et plus simple) des probabilités booléennes. Il est naturel d'introduire le concept de distributions $\eta$-diagonales, qui sont la contrepartie booléenne des distributions $R$-diagonales. Nous établissons un certain nombre de propriétés des distributions $\eta$-diagonales, avant d'examiner la bijection canonique reliant les distributions $\eta$ diagonales aux distributions $R$-diagonales indéfiniment divisibles. Le résultat principal est la paramétrisation par une paire de mesures boreliennes sur $[0, \infty)$ à support compact de toutes les lois $R$-diagonales $\boxplus$-indéfiniment divisibles pouvant apparaître dans un $C^{*}$-espace de probabilités. Parmi les applications de cette paramétrisation, nous montrons que l'ensemble des distributions $R$-diagonales $\boxplus$-indéfiniment divisibles est fermé sous l'opération $\nabla$ de convolution multiplicative libre.

MSC: Primary 46L54; secondary 46L53; 60E07; 60E10

Keywords: R-diagonal distribution; $\eta$-diagonal distribution; Free additive convolution; Infinite divisibility

\section{Introduction}

Free additive convolution $\boxplus$ is a binary operation on the set $\mathcal{P}$ of Borel probability measures on $\mathbb{R}$, reflecting the addition operation for free selfadjoint elements in a noncommutative probability space. The properties of this operation parallel in many respects the ones of the usual convolution on $\mathcal{P}$, for instance in the treatment of infinite divisibility.

\footnotetext{
${ }^{1}$ Supported in part by a grant from the National Science Foundation of the USA.

${ }^{2}$ Supported by a Discovery Grant from NSERC, Canada.
} 
One way to approach $\boxplus$-infinite divisibility is to use a bijection constructed in [5] which relates free independence to another form of noncommutative independence, namely Boolean independence. In this paper we focus on probability measures with compact support, so we view this bijection as a map $\mathbb{B}: \mathcal{P}_{c} \rightarrow \mathcal{P}_{c}^{\text {(inf-div) }}$, where $\mathcal{P}_{c}$ is the set of probability measures with compact support on $\mathbb{R}$, while $\mathcal{P}_{c}^{\text {(inf-div) }}$ consists of those measures $\mu \in \mathcal{P}_{c}$ which are $\boxplus$-infinitely divisible, that is, have the property that for every $n \in \mathbb{N}$, there exists $\mu_{n} \in \mathcal{P}_{c}$ satisfying

$$
\underbrace{\mu_{n} \boxplus \cdots \boxplus \mu_{n}}_{n}=\mu
$$

The bijection $\mathbb{B}$ connects the fundamental transforms of free and Boolean probability, the $R$-transform and respectively the $\eta$-series. For $\mu \in \mathcal{P}_{c}$, both of these transforms $R_{\mu}(z)$ and $\eta_{\mu}(z)$ are convergent power series. The bijection $\mathbb{B}$ is described by the equation

$$
R_{\mathbb{B}(\mu)}=\eta_{\mu}, \quad \mu \in \mathcal{P}_{c} .
$$

More precisely, for every $\mu \in \mathcal{P}_{c}$ there exists a uniquely determined measure $v \in \mathcal{P}_{c}^{\text {(inf-div) }}$ such that $R_{v}=\eta_{\mu}$, and one defines $\mathbb{B}(\mu):=v$.

At the level of compactly supported distributions, the bijection $\mathbb{B}$ is precisely the parametrization of $\boxplus$-infinitely divisible distributions provided in [19]. This was extended in [5] to the space $\mathcal{P}$ of all Borel probability measures on $\mathbb{R}$. In a different direction, the bijection $\mathbb{B}$ was extended in [3] to the space of joint distributions for $k$-tuples of selfadjoint elements in a $C^{*}$-probability space. Our goal in this paper is to use a multivariate version of the bijection $\mathbb{B}$ in order to study $\boxplus$-infinitely divisibile $R$-diagonal distributions, a significant class of $*$-distributions considered in free probability.

To explain our results, we introduce some notation. We let $\mathcal{D}_{c}(1, *)$ stand for the collection of all $*$-distributions of (generally, not selfadjoint) elements in a (generally not tracial) $C^{*}$-probability space. There is a natural operation $\boxplus$ on $\mathcal{D}_{c}(1, *)$ which corresponds to the addition $a+b$ of two variables $a, b$ in the same space such that $\left\{a, a^{*}\right\}$ is free from $\left\{b, b^{*}\right\}$. Infinite divisibility in $\mathcal{D}_{c}(1, *)$ is defined as in $(1.1)$, and we denote by $\mathcal{D}_{c}(1, *)^{\text {(inf-div) }}$ the collection of $\boxplus$-infinitely divisible elements of $\mathcal{D}_{c}(1, *)$. The notions of $R$-transform and $\eta$-series also have natural extensions to the context of $*$-distributions.

The results of [3], specialized to two selfadjoint variables, can be applied to $\mathcal{D}_{c}(1, *)$ after a simple change of coordinates. There is again a bijection $\mathbb{B}_{(1, *)}: \mathcal{D}_{c}(1, *) \rightarrow \mathcal{D}_{c}(1, *)^{\text {(inf-div) }}$ defined by the requirement that

$$
R_{\mathbb{B}_{(1, *)}(\mu)}=\eta_{\mu}, \quad \mu \in \mathcal{D}_{c}(1, *) .
$$

This is analogous to the condition (1.2) satisfied by the original bijection $\mathbb{B}$, but proving the existence of $\mathbb{B}_{(1, *)}$ is more than a trivial extension of the proof for $\mathbb{B}$, and requires a mixture of combinatorial and analytic methods.

We turn now to $R$-diagonal $*$-distributions, which can be succinctly described as the distributions in $\mathcal{D}_{c}(1, *)$ that are invariant under multiplication by a free Haar unitary (see [15, Theorem 15.10, p. 244]). This class of $*$-distributions has received quite a bit of attention in the free probability literature. In particular, elements with $R$ diagonal $*$-distributions were among the first examples of non-normal elements in $W^{*}$-probability spaces for which the so-caled 'Brown spectral measure' was calculated explicitly (in [10]), and for which the Brown measure techniques could be used to find invariant subspaces (in [17]). $R$-diagonal $*$-distributions also appear in large $N$ limit results for random matrices, in connection to the so-called 'single ring theorem' [9].

For our purposes, it is best to consider the original definition [14] of $R$-diagonal distributions which asks that the $R$-transform of the distribution be in some sense 'diagonal' [15, Definition 15.3, p. 241]. From this point of view, it is clear how to define the Boolean counterpart of $R$-diagonality: we simply say that a $*$-distribution is $\eta$-diagonal if its $\eta$-series is diagonal. The map $\mathbb{B}_{(1, *)}$ defined by (1.3) will then give a bijection between the set of all $\eta$-diagonal distributions in $\mathcal{D}_{c}(1, *)$ and the set of $R$-diagonal distributions in $\mathcal{D}_{c}(1, *)$ which are $\boxplus$-infinitely divisible.

The above discussion shows that there is some interest in studying $\eta$-diagonal distributions. In this paper we point out a few general algebraic and combinatorial properties of such a distribution $\mu$, which actually hold for $\mu$ in a larger, purely algebraic space $\mathcal{D}_{\mathrm{alg}}(1, *)$. The property of a distribution $\mu \in \mathcal{D}_{\mathrm{alg}}(1, *)$ of being $\eta$-diagonal has an elegant description phrased directly in terms of the $*$-moments of $\mu$. This result (Theorem 2.8) is reminiscent of 
(but simpler than) the description [13, Theorem 1.2.1] of $R$-diagonal variables in terms of their $*$-moments. The $\eta$ diagonal distributions also have other algebraic and combinatorial properties that are analogous to known properties of $R$-diagonal distributions. In particular, if $a$ is an $\eta$-diagonal element in a $*$-probability space $(\mathcal{A}, \varphi)$ (which means, by definition, that $a$ has $\eta$-diagonal $*$-distribution with respect to $\varphi$ ) then it follows that $a a^{*}$ and $a^{*} a$ are Boolean independent elements of $\mathcal{A}$, and that the coefficients of the $\eta$-series of $a a^{*}$ and $a^{*} a$ are read from the so-called determining sequences for the $*$-distribution of $a$. For details on the terms used above and for a discussion of why this is indeed analogous to known facts about $R$-diagonals, see Remark 3.4 below.

In the case in which the $\eta$-diagonal distribution $\mu$ is in $\mathcal{D}_{c}(1, *)$, we point out a natural parametrization for $\mu$, given by a pair of compactly supported Borel probability measures on $[0, \infty)$. That is, we establish a canonical bijection

$$
\left\{\mu \in \mathcal{D}_{c}(1, *): \mu \text { is } \eta \text {-diagonal }\right\} \ni \mu \leftrightarrow\left(\sigma_{1}, \sigma_{2}\right) \in \mathcal{P}_{c}^{+} \times \mathcal{P}_{c}^{+},
$$

where $\mathcal{P}_{c}^{+}:=\left\{\sigma \in \mathcal{P}_{c}: \sigma([0, \infty))=1\right\}$. Without going into details, we mention that all the $*$-distributions appearing in this paper are defined as linear functionals on the algebra $\mathbb{C}\left\langle Z, Z^{*}\right\rangle$ of complex polynomials in the non-commuting indeterminates $Z$ and $Z^{*}$, and that the correspondence $\mu \leftrightarrow\left(\sigma_{1}, \sigma_{2}\right)$ from (1.4) amounts to the equalities

$$
\mu\left(\left(Z Z^{*}\right)^{n}\right)=\int_{0}^{\infty} t^{n} d \sigma_{1}(t) \quad \text { and } \quad \mu\left(\left(Z^{*} Z\right)^{n}\right)=\int_{0}^{\infty} t^{n} d \sigma_{2}(t), \quad n \in \mathbb{N} .
$$

In other words, the probability measures $\sigma_{1}$ and $\sigma_{2}$ which parametrize $\mu$ in (1.4) are simply the distributions of $Z Z^{*}$ and of $Z^{*} Z$ with respect to the functional $\mu$. The relevant point here is that for any given $\sigma_{1}, \sigma_{2} \in \mathcal{P}_{c}^{+}$there exists a unique $\eta$-diagonal distribution $\mu \in \mathcal{D}_{c}(1, *)$ such that $(1.5)$ holds; we will prove this point by producing an operator model for $\mu$ (an operator $A$ on a Hilbert space $\mathcal{K}$, such that the required $\mu$ appears as $*$-distribution of $A$ with respect to a suitably chosen functional on $B(\mathcal{K})$ ).

When the bijection $\mathbb{B}_{(1, *)}$ is applied to $\left\{\mu \in \mathcal{D}_{c}(1, *): \mu\right.$ is $\eta$-diagonal $\}$ in (1.4), we obtain a bijection

$$
\left\{v \in \mathcal{D}_{c}(1, *): \begin{array}{l}
v \text { is } R \text {-diagonal and } \\
\boxplus \text {-infinitely divisible }
\end{array}\right\} \ni v \leftrightarrow\left(\sigma_{1}, \sigma_{2}\right) \in \mathcal{P}_{c}^{+} \times \mathcal{P}_{c}^{+}
$$

Thus, we have a parametrization of a general $\boxplus$-infinitely divisible $R$-diagonal distribution by a pair of probability measures from $\mathcal{P}_{c}^{+}$. Analogously to (1.5), it is possible to write explicitly the relation connecting $\sigma_{1}, \sigma_{2}$ to the distributions of the elements $Z Z^{*}$ and $Z^{*} Z$ in the noncommutative probability space $\left(\mathbb{C}\left\langle Z, Z^{*}\right\rangle, v\right)$. Theorem 6.4 below realizes this parametrization by providing precise formulas for the $R$-transforms of $Z Z^{*}$ and of $Z^{*} Z$.

An important subclass of $R$-diagonal distributions consists of those which satisfy the KMS condition for some parameter $t \in(0, \infty)$. This is a generalization of the trace condition, where the latter corresponds to the special case $t=1$ (see the review in Section 3 below). For an $R$-diagonal distribution $v$ which satisfies KMS with parameter $t$, one can process further the result of Theorem 6.4 in order to obtain explicit formulas for the distributions of $Z Z^{*}$ and of $Z^{*} Z$, in terms of the probability measures $\sigma_{1}$ and $\sigma_{2}$ which parametrize $v$. These formulas invoke some commonly used elements of free harmonic analysis on $\mathcal{P}_{c}^{+}$, and are given in Proposition 6.8.

As an application of the parametrization from (1.6), we prove that the set of $\boxplus$-infinitely divisible $R$-diagonal $*$-distributions is closed under the operation $\otimes$ of free multiplicative convolution. This provides a non-selfadjoint counterpart to the line of studying closure properties of $\boxplus$-infinitely divisible distributions which was pursued in the selfadjoint framework in [1] (also, quite recently, in [8]).

In addition to the present Introduction, the paper contains 6 sections. Section 2 introduces $\eta$-diagonal $*$-distributions and discusses some of their algebraic properties. Section 3 is devoted to a review of $R$-diagonal $*$-distributions, with emphasis on facts that are needed in the present paper. In Section 4 we verify that the bijection $\mathbb{B}_{(1, *)}$ does indeed work on $\mathcal{D}_{c}(1, *)$ in the way described in (1.3). Section 5 presents the operator model for $\eta$-diagonals which is the main ingredient in establishing the bijection (1.4). Section 6 contains our results concerning the parametrization of infinitely divisible $R$-diagonal distributions, and a discussion of the KMS example. Finally, Section 7 discusses the application to free multiplicative convolution. 


\section{2. $\eta$-series and $\eta$-diagonal $*$-distributions}

Notation 2.1. (1) We denote by $\mathcal{W}^{+}$the set $\bigsqcup_{n=1}^{\infty}\{1, *\}^{n}$ consisting of all non-empty words over the two-letter alphabet $\{1, *\}$. This is a semigroup (without unit) under the natural operation of concatenation. We denote by $|w|$ the number of letters in a word $w \in \mathcal{W}^{+}$.

(2) The algebra of complex polynomials in two non-commuting variables $Z$ and $Z^{*}$ is denoted, as usual, by $\mathbb{C}\left\langle Z, Z^{*}\right\rangle$. For every word $w=\left(\ell_{1}, \ldots, \ell_{n}\right) \in \mathcal{W}^{+}$we write

$$
Z^{w}=Z^{\ell_{1}} \cdots Z^{\ell_{n}} \in \mathbb{C}\left\langle Z, Z^{*}\right\rangle
$$

The set $\{1\} \cup\left\{Z^{w}: w \in \mathcal{W}^{+}\right\}$is a basis of $\mathbb{C}\left\langle Z, Z^{*}\right\rangle$ as a complex vector space.

(3) An algebraic $*$-distribution is a linear functional $\mu: \mathbb{C}\left\langle Z, Z^{*}\right\rangle \rightarrow \mathbb{C}$ such that $\mu(1)=1$. (At this stage we do not require $\mu$ to have any additional properties.) The values of $\mu$ on monomials $Z^{w}$ (with $w \in \mathcal{W}^{+}$) will be referred to as $*$-moments of $\mu$.

(4) The collection of all algebraic $*$-distributions from (3) is denoted $\mathcal{D}_{\text {alg }}(1, *)$.

Notation 2.2 (Series and their coefficients). (1) The algebra of formal power series in two non-commuting indeterminates $z$ and $z^{*}$ is denoted, as usual, by $\mathbb{C}\left\langle\left\langle z, z^{*}\right\rangle\right\rangle$. The collection $\mathbb{C}_{0}\left\langle\left\langle z, z^{*}\right\rangle\right\rangle \subset \mathbb{C}\left\langle\left\langle z, z^{*}\right\rangle\right\rangle$ of power series with vanishing constant coefficient is a two-sided ideal in $\mathbb{C}\left\langle\left\langle z, z^{*}\right\rangle\right\rangle$. An arbitrary element $f \in \mathbb{C}_{0}\left\langle\left\langle z, z^{*}\right\rangle\right\rangle$ is of the form

$$
f\left(z, z^{*}\right)=\sum_{n=1}^{\infty} \sum_{\ell_{1}, \ldots, \ell_{n} \in\{1, *\}} \alpha_{\left(\ell_{1}, \ldots, \ell_{n}\right)} z^{\ell_{1}} \cdots z^{\ell_{n}}=\sum_{w \in \mathcal{W}^{+}} \alpha_{w} z^{w},
$$

where the coefficients $\alpha_{w}$ are complex numbers, and for $w=\left(\ell_{1}, \ldots, \ell_{n}\right) \in \mathcal{W}^{+}$we use the notation $z^{w}=z^{\ell_{1}} \cdots z^{\ell_{n}}$

(2) Given $w \in \mathcal{W}^{+}$, we denote by $\mathrm{Cf}_{w}: \mathbb{C}_{0}\left\langle\left\langle z, z^{*}\right\rangle\right\rangle \rightarrow \mathbb{C}$ the linear functional which extracts the coefficient of $z^{w}$ from a series $f \in \mathbb{C}_{0}\left\langle\left\langle z, z^{*}\right\rangle\right\rangle$. That is, if $f$ is given by (2.1), we have $\mathrm{Cf}_{w}(f)=\alpha_{w}, w \in \mathcal{W}^{+}$.

(3) It will be convenient to also have a dedicated notation for certain products made with the linear functionals $\mathrm{Cf}_{w}$ from the preceding paragraph. More precisely, let $n$ be a positive integer and let $\pi=\left\{B_{1}, \ldots, B_{k}\right\}$ be a partition of $\{1, \ldots, n\}$; the latter term means that $B_{1}, \ldots, B_{k}$ (which are called the blocks of $\pi$ ) are non-empty sets with $B_{i} \cap B_{j}=$ $\varnothing$ for $i \neq j$ and with $B_{1} \cup \ldots \cup B_{k}=\{1, \ldots, n\}$. Given a word $w=\left(\ell_{1}, \ldots, \ell_{n}\right) \in \mathcal{W}^{+}$with $|w|=n$, we then define a functional (non-linear unless $\pi$ consists of only one block) $\mathrm{Cf}_{w ; \pi}: \mathbb{C}_{0}\left\langle\left\langle z, z^{*}\right\rangle\right\rangle \rightarrow \mathbb{C}$, as follows. For every block $B_{i}=\left\{b_{i, 1}, \ldots, b_{i, m}\right\}$ where $1 \leq b_{i, 1}<\cdots<b_{i, m} \leq n$, we set

$$
w\left|B_{i}=\left(\ell_{1}, \ldots, \ell_{n}\right)\right| B_{i}:=\left(\ell_{b_{i, 1}}, \ldots, \ell_{b_{i, m}}\right) \in\{1, *\}^{m} .
$$

Then we define

$$
\mathrm{Cf}_{w ; \pi}(f):=\prod_{i=1}^{k} \mathrm{Cf}_{w \mid B_{i}}(f), \quad f \in \mathbb{C}_{0}\left\langle\left\langle z, z^{*}\right\rangle\right\rangle .
$$

[Suppose, for instance, that $n=5, \pi=\{\{1,4,5\},\{2,3\}\}$, and $w=\left(\ell_{1}, \ldots, \ell_{5}\right)$. Then $\mathrm{Cf}_{w ; \pi}(f)=\mathrm{Cf}_{\left(\ell_{1}, \ell_{4}, \ell_{5}\right)}(f)$. $\left.\mathrm{Cf}_{\left(\ell_{2}, \ell_{3}\right)}(f), f \in \mathbb{C}_{0}\left\langle\left\langle z, z^{*}\right\rangle\right\rangle.\right]$

Definition and Remark 2.3 (Moment series, $\eta$-series). Fix $\mu \in \mathcal{D}_{\mathrm{alg}}(1, *)$. (1) The moment series of $\mu$ is defined as $M_{\mu}:=\sum_{w \in \mathcal{W}^{+}} \mu\left(Z^{w}\right) z^{w} \in \mathbb{C}_{0}\left\langle\left\langle z, z^{*}\right\rangle\right\rangle$.

(2) The $\eta$-series of $\mu$ is defined as

$$
\eta_{\mu}:=M_{\mu}\left(1+M_{\mu}\right)^{-1}=\left(1+M_{\mu}\right)^{-1} M_{\mu} \in \mathbb{C}_{0}\left\langle\left\langle z, z^{*}\right\rangle\right\rangle,
$$

where all the algebraic operations are performed in the algebra $\mathbb{C}\left\langle\left\langle z, z^{*}\right\rangle\right\rangle$. 
(3) It is immediate from (2.2) that the series $M_{\mu}$ can be retrieved from $\eta_{\mu}$ by the formula

$$
M_{\mu}=\eta_{\mu}\left(1-\eta_{\mu}\right)^{-1}=\left(1-\eta_{\mu}\right)^{-1} \eta_{\mu} .
$$

(4) The right-hand side of (2.3) can be written as a geometric series $\sum_{k=1}^{\infty} \eta_{\mu}^{k}$, which converges coefficientwise, in the sense that for every $w \in \mathcal{W}^{+}$one has

$$
\mathrm{Cf}_{w}\left(M_{\mu}\right)=\sum_{k=1}^{\infty} \mathrm{Cf}_{w}\left(\eta_{\mu}^{k}\right) \quad \text { (convergence in } \mathbb{C} \text { ). }
$$

The series on the right-hand side of the latter equation is convergent due to the obvious reason that, denoting $|w|=: n$, one has $\mathrm{Cf}_{w}\left(\eta_{\mu}^{k}\right)=0$ for all $k>n$. It is also worth recording that for $1 \leq k \leq n$ the coefficient $\mathrm{Cf}_{w}\left(\eta_{\mu}^{k}\right)$ can be related (via a direct inspection of how the $k$-fold product of $\eta_{\mu}$ with itself is formed) to the generalized coefficients $\mathrm{Cf}_{w ; \pi}$ from Notation 2.2:

$$
\mathrm{Cf}_{w}\left(\eta_{\mu}^{k}\right)=\sum_{\substack{\pi \text { interval partition } \\ \text { with } k \text { blocks of }\{1, \ldots, n\}}} \mathrm{Cf}_{w ; \pi}\left(\eta_{\mu}\right) .
$$

In (2.4) we used the customary term of interval partition for a partition $\pi$ of $\{1, \ldots, n\}$ such that every block $B$ of $\pi$ is of the form $\{a, a+1, \ldots, b\}$ for some $a \leq b$ in $\{1, \ldots, n\}$. We will also use the customary notation Int $(n)$ for the set of all interval partitions of $\{1, \ldots, n\}$. By summing over $1 \leq k \leq n$ in equation (2.4), one obtains a very useful explicit formula for the coefficients of $M_{\mu}$ in terms of those of $\eta_{\mu}$, namely

$$
\mathrm{Cf}_{w}\left(M_{\mu}\right)=\sum_{\pi \in \operatorname{Int}(n)} \mathrm{Cf}_{w ; \pi}\left(\eta_{\mu}\right), \quad w \in \mathcal{W}^{+} \text {with }|w|=n .
$$

An analogous argument converts (2.2) into the formula

$$
\mathrm{Cf}_{w}\left(\eta_{\mu}\right)=\sum_{\pi \in \operatorname{Int}(n)}(-1)^{1+|\pi|} \mathrm{Cf}_{w ; \pi}\left(M_{\mu}\right), \quad w \in \mathcal{W}^{+} \text {with }|w|=n
$$

where $|\pi|$ denotes the number of blocks of the partition $\pi$.

Remark 2.4. It is clear that the map $\mathcal{D}_{\text {alg }}(1, *) \ni \mu \mapsto M_{\mu} \in \mathbb{C}_{0}\left\langle\left\langle z, z^{*}\right\rangle\right\rangle$ is bijective. Equations (2.2) and (2.3) show that the map $\mathcal{D}_{\text {alg }}(1, *) \ni \mu \mapsto \eta_{\mu} \in \mathbb{C}_{0}\left\langle\left\langle z, z^{*}\right\rangle\right\rangle$ is a bijection as well. In other words, we can define a distribution $\mu \in \mathcal{D}_{\text {alg }}(1, *)$ by specifying its $\eta$-series.

Definition 2.5. A word $w \in \mathcal{W}^{+}$is said to be alternating when it is of the form

$$
w=(\underbrace{1, *, 1, *, \ldots, 1, *}_{2 m})=(1, *)^{m} \quad \text { or } \quad w=(\underbrace{*, 1, *, 1, \ldots, *, 1}_{2 m})=(*, 1)^{m},
$$

for some positive integer $m$. In the first case $w$ is said to be of type $(1, *)$, and in the second case $w$ is said to be of type $(*, 1)$. In these formulas, powers are taken relative to concatenation. Note in particular that alternating words have positive, even length.

Definition 2.6. (1) A distribution $\mu \in \mathcal{D}_{\text {alg }}(1, *)$ is said to be $\eta$-diagonal if $\mathrm{Cf}_{w}\left(\eta_{\mu}\right)=0$ for every word $w \in \mathcal{W}^{+}$ which is not alternating.

(2) If $\mu$ is $\eta$-diagonal, its $\eta$-series is thus of the form

$$
\eta_{\mu}\left(z, z^{*}\right)=\sum_{n=1}^{\infty} \alpha_{n}\left(z z^{*}\right)^{n}+\sum_{n=1}^{\infty} \beta_{n}\left(z^{*} z\right)^{n}
$$


with $\alpha_{n}, \beta_{n} \in \mathbb{C}$ for $n \in \mathbb{N}$. The sequences $\left(\alpha_{n}\right)_{n=1}^{\infty}$ and $\left(\beta_{n}\right)_{n=1}^{\infty}$ will be called the determining sequences of the $\eta$-diagonal distribution $\mu$.

The main goal of the present section is to reveal an equivalent characterization of $\eta$-diagonal distributions, which is phrased directly in terms of $*$-moments. For this purpose, we require one more concept related to words in $\mathcal{W}^{+}$.

Definition and Remark 2.7. (1) A word $w=\left(\ell_{1}, \ldots, \ell_{n}\right) \in \mathcal{W}^{+}$is said to be mixed-alternating if $n=2 m$ is even and if the letters of $w$ are such that $\ell_{2 k-1} \neq \ell_{2 k}$, for $k=1,2, \ldots, m$. Equivalently, $w$ is mixed-alternating when it belongs to the concatenation subsemigroup of $\mathcal{W}^{+}$generated by the words $(1, *)$ and $(*, 1)$.

(2) By grouping factors, it is easily seen that every mixed-alternating word $w$ can be written in a unique way as a concatenation of alternating words such that consecutive words are of different types. Indeed, if we write such a word as

$$
w=w_{1} w_{2} \cdots w_{p}
$$

where $p \geq 1$, each $w_{i}$ is alternating, and $w_{i}$ is not of the same type as $w_{i+1}, i=1,2, \ldots, p-1$, then the boundaries between the words $w_{1}, \ldots, w_{p}$ can be retrieved at the places where $w$ has two consecutive identical letters.

[For example, $w=(1, *, 1, *, 1, *, *, 1, *, 1,1, *, *, 1, *, 1)$ is mixed-alternating and its canonical factorization (2.7) is $w_{1} w_{2} w_{3} w_{4}$ with $w_{1}=(1, *)^{3}, w_{2}=(*, 1)^{2}, w_{3}=(1, *), w_{4}=(*, 1)^{2}$.]

Theorem 2.8. For every distribution $\mu \in \mathcal{D}_{\mathrm{alg}}(1, *)$, the statements (a) and (b) are equivalent.

(a) $\mu$ is $\eta$-diagonal.

(b) $\mu$ satisfies the following conditions ${ }^{3}$ :

( $\eta \mathrm{DM} 1)$ Whenever $w \in \mathcal{W}^{+}$is not mixed-alternating, it follows that $\mu\left(Z^{w}\right)=0$.

( $\eta \mathrm{DM} 2)$ Whenever $w=w_{1} \cdots w_{p} \in \mathcal{W}^{+}$is mixed-alternating and factored as in (2.7), it follows that $\mu\left(Z^{w}\right)=$ $\mu\left(Z^{w_{1}}\right) \cdots \mu\left(Z^{w_{p}}\right)$.

The proof of the implication (b) $\Rightarrow$ (a) in the above theorem requires two auxilliary results.

Lemma 2.9. Suppose that a distribution $\mu \in \mathcal{D}_{\mathrm{alg}}(1, *)$ satisfies the condition $(\eta \mathrm{DM} 1)$. Then $\mathrm{Cf}_{w}\left(\eta_{\mu}\right)=0$ for every word $w \in \mathcal{W}^{+}$that is not mixed-alternating.

Proof. Suppose that $w$ is not mixed alternating and $|w|=n$. We prove that $\mathrm{Cf}_{w}\left(\eta_{\mu}\right)=0$ by showing that each term in the right-hand side of (2.6) vanishes. Indeed, let $\pi=\left\{J_{1}, \ldots, J_{m}\right\} \in \operatorname{Int}(n)$ be a partition where the intervals $J_{1}, \ldots, J_{m}$ are listed in increasing order. Observe that $w=\left(w \mid J_{1}\right) \cdots\left(w \mid J_{m}\right)$ (concatenation product). Since $w$ is not mixed-alternating, there must exist an index $1 \leq k \leq m$ such that $w \mid J_{k}$ is not mixed-alternating. For this $k$, condition ( $\eta \mathrm{DM} 1$ ) yields $\mathrm{Cf}_{w \mid J_{k}}\left(M_{\mu}\right)=0$. Therefore the term indexed by $\pi$ in (2.6) vanishes as well because $\mathrm{Cf}_{w \mid J_{k}}\left(M_{\mu}\right)$ is one of its factors. The lemma follows.

Lemma 2.10. Let $\mu, v \in \mathcal{D}_{\mathrm{alg}}(1, *)$ be such that

(1) Both $\mu$ and $v$ satisfy conditions ( $\eta \mathrm{DM} 1)$ and ( $\eta \mathrm{DM} 2)$, and

(2) $\mathrm{Cf}_{w}\left(\eta_{\mu}\right)=\mathrm{Cf}_{w}\left(\eta_{\nu}\right)$ for every alternating word $w \in \mathcal{W}^{+}$.

Then $\mu=v$.

Proof. If $w \in \mathcal{W}^{+}$is not mixed-alternating then $\mu\left(Z^{w}\right)=v\left(Z^{w}\right)=0$ because $\mu$ and $v$ satisfy ( $\eta$ DM1). Thus it suffices to verify that $\mu\left(Z^{w}\right)=v\left(Z^{w}\right)$ for mixed-alternating words $w$. In fact, it suffices to prove this equality when

\footnotetext{
${ }^{3}$ The acronym $\eta \mathrm{DM}$ is meant to suggest $\eta$-Diagonality-in-Moments.
} 
$w$ is alternating. Indeed, suppose for the moment that the equality has been proved for alternating words and let $w \in \mathcal{W}^{+}$be a mixed-alternating word. Consider the canonical factorization $w=w_{1} \cdots w_{p}$ indicated in (2.7). We have

$$
\begin{aligned}
\mu\left(Z^{w}\right) & =\mu\left(Z^{w_{1}}\right) \cdots \mu\left(Z^{w_{p}}\right) \quad(\text { by }(\eta \mathrm{DM} 2) \text { for } \mu) \\
& =v\left(Z^{w_{1}}\right) \cdots v\left(Z^{w_{p}}\right) \quad(\text { by assumption on alternating moments) } \\
& =v\left(Z^{w}\right) \quad(\text { by }(\eta \mathrm{DM} 2) \text { for } v) .
\end{aligned}
$$

We conclude the proof by showing that $\mu\left(Z^{w}\right)=v\left(Z^{w}\right)$ for every alternating word $w$. By symmetry, it suffices to verify that $\mu\left(\left(Z^{*} Z\right)^{m}\right)=v\left(\left(Z^{*} Z\right)^{m}\right)$ for every $m \in \mathbb{N}$. Fix $m$ and write $\mu\left(\left(Z^{*} Z\right)^{m}\right)$ and $v\left(\left(Z^{*} Z\right)^{m}\right)$ as sums indexed by $\operatorname{Int}(2 m)$, in the way indicated in (2.5). We show that for every $\pi \in \operatorname{Int}(2 m)$, the terms indexed by $\pi$ in the two sums (for $\mu$ and for $\nu$ ) are equal to each other. If $\pi$ has a block $B$ of odd cardinality, then the terms we are looking at are both equal to 0 because they include the factors $\mathrm{Cf}_{w \mid B}\left(\eta_{\mu}\right)$ and respectively $\mathrm{Cf}_{w \mid B}\left(\eta_{v}\right)$, and these factors are zero by Lemma 2.9. If all the blocks of $\pi$ are even, we write $\pi=\left\{J_{1}, \ldots, J_{k}\right\}$ where the intervals $J_{1}, \ldots, J_{k}$ are listed in increasing order, and where $\left|J_{1}\right|=2 d_{1}, \ldots,\left|J_{k}\right|=2 d_{k}$ for some $d_{1}, \ldots, d_{k} \in \mathbb{N}$. The terms indexed by $\pi$ in the two sums we consider are then

$$
\prod_{i=1}^{k} \mathrm{Cf}_{(*, 1)^{d_{i}}}\left(\eta_{\mu}\right) \text { and respectively } \prod_{i=1}^{k} \mathrm{Cf}_{(*, 1)^{d_{i}}}\left(\eta_{\nu}\right),
$$

where we used the fact that $(*, 1)^{m} \mid J_{i}=(*, 1)^{d_{i}}$, for $1 \leq i \leq k$. The two products in (2.8) are indeed equal by assumption (2) in the statement.

Proof of Theorem 2.8. Suppose first that $\mu$ is $\eta$-diagonal. We verify that it satisfies ( $\eta$ DM1) and ( $\eta$ DM2). The hypothesis on $\mu$ says in particular that $\mathrm{Cf}_{w}\left(\eta_{\mu}\right)=0$ for every $w \in \mathcal{W}^{+}$which is not mixed-alternating. To prove ( $\eta \mathrm{DM} 1$ ), we must show that $\mathrm{Cf}_{w}\left(M_{\mu}\right)=0$ for every such word. This argument is carried precisely as in the proof of Lemma 2.9, with the roles of $M_{\mu}$ and $\eta_{\mu}$ being reversed and with (2.5) in place of (2.6). The reader will have no difficulty verifying the details.

In order to show that $\mu$ also satisfies ( $\eta \mathrm{DM} 2)$, fix a mixed-alternating word $w \in \mathcal{W}^{+}$, with canonical factorization $w=w_{1} \cdots w_{p}$ as in (2.7). Set $n=|w|=\left|w_{1}\right|+\cdots+\left|w_{p}\right|$, and let $\rho_{0}$ be the partition of $\{1, \ldots, n\}$ into intervals $J_{1}, \ldots, J_{p}$ (written in increasing order) with lengths $\left|J_{1}\right|=\left|w_{1}\right|, \ldots,\left|J_{p}\right|=\left|w_{p}\right|$. Given a partition $\pi$ of $\{1, \ldots, n\}$, we write $\pi \leq \rho_{0}$ if every block $B$ of $\pi$ is contained in one of the blocks $J_{1}, \ldots, J_{p}$ of $\rho_{0}$. (This relation is usually called the reverse refinement order on partitions.)

Next, we use (2.5) to express the coefficient $\mathrm{Cf}_{w}\left(M_{\mu}\right)=\mu\left(Z^{w}\right)$ as a sum indexed by $\operatorname{Int}(n)$. The special structure of the coefficients of $\eta_{\mu}$ implies that a partition $\pi \in \operatorname{Int}(n)$ has a zero contribution to that sum unless $\pi \leq \rho_{0}$. It is immediate that the partitions $\pi \in \operatorname{Int}(n)$ satisfying $\pi \leq \rho_{0}$ are in natural bijective correspondence to tuples of partitions $\left(\pi_{1}, \ldots, \pi_{p}\right)$ where $\pi_{1} \in \operatorname{Int}\left(J_{1}\right), \ldots, \pi_{p} \in \operatorname{Int}\left(J_{p}\right)$. This correspondence is such that for $\pi \leftrightarrow\left(\pi_{1}, \ldots, \pi_{p}\right)$ we have

$$
\mathrm{Cf}_{w ; \pi}\left(\eta_{\mu}\right)=\mathrm{Cf}_{w_{1} ; \pi_{1}}\left(\eta_{\mu}\right) \cdots \mathrm{Cf}_{w_{p} ; \pi_{p}}\left(\eta_{\mu}\right) .
$$

These observations lead to the formula

$$
\mu\left(Z^{w}\right)=\sum_{\substack{\pi_{1} \in \operatorname{Int}\left(J_{1}\right), \ldots ., \pi_{p} \in \operatorname{Int}\left(J_{p}\right)}} \mathrm{Cf}_{w_{1} ; \pi_{1}}\left(\eta_{\mu}\right) \cdots \mathrm{Cf}_{w_{p} ; \pi_{p}}\left(\eta_{\mu}\right)=\prod_{i=1}^{p}\left(\sum_{\pi_{i} \in \operatorname{Int}\left(J_{i}\right)} \mathrm{Cf}_{w_{i} ; \pi_{i}}\left(\eta_{\mu}\right)\right) .
$$

In the latter product, one more application of (2.5) identifies

$$
\sum_{\pi_{i} \in \operatorname{Int}\left(J_{i}\right)} \mathrm{Cf}_{w_{i} ; \pi_{i}}\left(\eta_{\mu}\right)=\mu\left(Z^{w_{i}}\right), \quad 1 \leq i \leq p,
$$

thus implying the desired conclusion that $\mu\left(Z^{w}\right)=\prod_{i=1}^{p} \mu\left(Z^{w_{i}}\right)$. 
Conversely, assume now that $\mu$ satisfies conditions ( $\eta$ DM1) and ( $\eta$ DM2). We show that it is $\eta$-diagonal by an indirect argument: we construct an $\eta$-diagonal distribution $v \in \mathcal{D}_{\text {alg }}(1, *)$ and then prove that $\mu=v$. The distribution $v$ is defined by specifying its $\eta$-series (see Remark 2.4), namely $\mathrm{Cf}_{w}\left(\eta_{\nu}\right)=\mathrm{Cf}_{w}\left(\eta_{\mu}\right)$ if $w$ is alternating and $\mathrm{Cf}_{w}\left(\eta_{\nu}\right)=0$ otherwise. To prove that $\mu=v$ we show that $\mu$ and $v$ satisfy the hypothesis of Lemma 2.10. Indeed, both $\mu$ and $v$ satisfy ( $\eta$ DM1) and ( $\eta$ DM2): $\mu$ does so by hypothesis, while $v$ does so because it is $\eta$-diagonal and by virtue of the implication (a) $\Rightarrow$ (b) proved above. On the other hand, if $w \in \mathcal{W}^{+}$is an alternating word, the equality $\mathrm{Cf}_{w}\left(\eta_{\mu}\right)=$ $\mathrm{Cf}_{w}\left(\eta_{v}\right)$ is true by the definition of $v$. This concludes the proof of the theorem.

Remark 2.11. Let $(\mathcal{A}, \varphi)$ be a noncommutative probability space (that is, $\mathcal{A}$ is a unital algebra over $\mathbb{C}, \varphi: \mathcal{A} \rightarrow \mathbb{C}$ is a linear functional, and $\varphi(1)=1$ ), and let $a_{1}, a_{2} \in \mathcal{A}$. Recall [18] that $a_{1}, a_{2}$ are said to be Boolean independent provided that, given positive integers $n, p_{1}, \ldots, p_{n}$ and indices $i_{1}, \ldots, i_{n} \in\{1,2\}$ such that $i_{k} \neq i_{k+1}$ for $i=1, \ldots, n-1$, the following identity is satisfied:

$$
\varphi\left(a_{i_{1}}^{p_{1}} \cdots a_{i_{n}}^{p_{n}}\right)=\varphi\left(a_{i_{1}}^{p_{1}}\right) \cdots \varphi\left(a_{i_{n}}^{p_{n}}\right) .
$$

Now consider the noncommutative probability space $\left(\mathbb{C}\left\langle Z, Z^{*}\right\rangle, \mu\right)$, where $\mu \in \mathcal{D}_{\mathrm{alg}}(1, *)$ is $\eta$-diagonal. Condition ( $\eta \mathrm{DM} 2$ ) of Theorem 2.8 can be restated as saying that $Z Z^{*}$ and $Z^{*} Z$ are Boolean independent in $\left(\mathbb{C}\left\langle Z, Z^{*}\right\rangle, \mu\right)$.

Remark and Notation 2.12. Another relevant fact concerning an $\eta$-diagonal $*$-distribution $\mu$ concerns the individual $\eta$-series of $Z Z^{*}$ and $Z^{*} Z$ in the noncommutative probability space $\left(\mathbb{C}\left\langle Z, Z^{*}\right\rangle, \mu\right)$. If $a$ is an element in a noncommutative probability space $(\mathcal{A}, \varphi)$, then its moment series and $\eta$-series $M_{a}, \eta_{a} \in \mathbb{C} \llbracket z \rrbracket$ are defined as in Definition 2.3 but using moments in place of $*$-moments: first we set $M_{a}(z)=\sum_{n=1}^{\infty} \varphi\left(a^{n}\right) z^{n}$, and then define

$$
\eta_{a}(z)=M_{a}(z) /\left(1+M_{a}(z)\right) \in \mathbb{C} \llbracket z \rrbracket .
$$

The coefficients of $M_{a}$ and $\eta_{a}$ are related to each other via summations over interval partitions which are analogous to those shown in (2.5), (2.6) (and are derived the same way, by starting from the algebraic relations satisfied by the series themselves). We explicitly record here the analogue of (2.6):

$$
\mathrm{Cf}_{n}\left(\eta_{a}\right)=\sum_{\rho \in \operatorname{Int}(n)}(-1)^{1+|\rho|} \prod_{B \in \rho} \varphi\left(a^{|B|}\right), \quad n \in \mathbb{N},
$$

where (by analogy with Notation 2.2(2)) we use the notation $\mathrm{Cf}_{n}: \mathbb{C} \llbracket z \rrbracket \rightarrow \mathbb{C}$ for the linear map that extracts the $n$th coefficient of a series in $\mathbb{C} \llbracket z \rrbracket$.

When applied to the elements $Z Z^{*}$ and $Z^{*} Z$ from the framework of Theorem 2.8, these observations yield the following result.

Proposition 2.13. Suppose that $\mu \in \mathcal{D}_{\mathrm{alg}}(1, *)$ is an $\eta$-diagonal distribution, and let $\left(\alpha_{n}\right)_{n=1}^{\infty},\left(\beta_{n}\right)_{n=1}^{\infty}$ be its determining sequences (as introduced in Definition 2.6(2)). Then in the noncommutative probability space $\left(\mathbb{C}\left\langle Z, Z^{*}\right\rangle, \mu\right)$, the elements $Z Z^{*}$ and $Z^{*} Z$ have $\eta$-series given by

$$
\eta_{Z Z^{*}}(z)=\sum_{n=1}^{\infty} \alpha_{n} z^{n} \quad \text { and } \quad \eta_{Z^{*} Z}(z)=\sum_{n=1}^{\infty} \beta_{n} z^{n} .
$$

Proof. By symmetry, it suffices to prove the first formula. Equation (2.9) yields

$$
\mathrm{Cf}_{n}\left(\eta_{Z Z^{*}}\right)=\sum_{\rho \in \operatorname{Int}(n)}(-1)^{1+|\rho|} \prod_{B \in \rho} \mu\left(\left(Z Z^{*}\right)^{|B|}\right), \quad n \in \mathbb{N} .
$$

For the remainder of the proof, we fix $n \in \mathbb{N}$ and verify that the right-hand side of (2.10) is equal to $\alpha_{n}$.

For every partition $\rho=\left\{J_{1}, \ldots, J_{k}\right\} \in \operatorname{Int}(n)$, with intervals $J_{1}, \ldots, J_{k}$ written in increasing order, we define a doubled partition $\widehat{\rho}=\left\{\widehat{J}_{1}, \ldots, \widehat{J}_{k}\right\} \in \operatorname{Int}(2 n)$. This is the interval partition uniquely determined by the requirement 
that $\widehat{J}_{1}, \ldots, \widehat{J}_{k}$ come in increasing order and satisfy $\left|\widehat{J}_{i}\right|=2\left|J_{i}\right|$ for $1 \leq i \leq k$. With this notation, it is easily seen that the right-hand side of (2.10) can be written as

$$
\sum_{\rho \in \operatorname{Int}(n)}(-1)^{1+|\widehat{\rho}|} \mathrm{Cf}_{(1, *)^{n} ; \widehat{\rho}}\left(M_{\mu}\right)
$$

This, however, is the same as

$$
\sum_{\pi \in \operatorname{Int}(2 n)}(-1)^{1+|\pi|} \operatorname{Cf}_{(1, *)^{n} ; \pi}\left(M_{\mu}\right)
$$

Indeed, due to the special structure of the $*$-moments of $\mu$ described in Theorem 2.8 , all the terms in the latter sum, corresponding to partitions $\pi \in \operatorname{Int}(2 n)$ which are not of the form $\widehat{\rho}$, are equal to 0 . We conclude that

$$
\mathrm{Cf}_{n}\left(\eta_{Z Z^{*}}\right)=\sum_{\pi \in \operatorname{Int}(2 n)}(-1)^{1+|\pi|} \operatorname{Cf}_{(1, *)^{n} ; \pi}\left(M_{\mu}\right)=\operatorname{Cf}_{(1, *)^{n}}\left(\eta_{\mu}\right)=\alpha_{n},
$$

where (2.6) is used in the second equality.

\section{3. $R$-transforms and $R$-diagonal $*$-distributions}

The discussion in Section 2 is better put into perspective when one compares it to the parallel (more elaborate) free probability framework. In the free probability framework, instead of $\eta$-series one works with $R$-transforms, and one has the concept of what it means for a $*$-distribution $\mu \in \mathcal{D}_{\text {alg }}(1, *)$ to be $R$-diagonal. The class of $R$-diagonal $*$-distributions is in fact rather well-studied in the free probability literature. Here we review some of their basic properties, mostly following [15, Lecture 15], and with emphasis on the facts we need in the present work.

Remark 3.1. On a combinatorial level, switching to the world of free probability comes to using non-crossing partitions instead of interval partitions. We recall that a crossing of a partition $\pi$ of $\{1, \ldots, n\}$ consists of integers $1 \leq a<b<c<d \leq n$ such that the set $\{a, c\}$ is contained in a block of $\pi$ and $\{b, d\}$ is contained in a different block of $\pi$. A partition is non-crossing if it has no crossings. We denote by $N C(n)$ the collection of all non-crossing partitions of $\{1, \ldots, n\}$.

Similarly to the lattice $\operatorname{Int}(n)$, the set $N C(n)$ is partially ordered by reverse refinement. The minimal and maximal elements with respect to this partial order are denoted by $0_{n}$ (the partition of $\{1, \ldots, n\}$ into $n$ singleton blocks) and respectively $1_{n}$ (the partition of $\{1, \ldots, n\}$ into one block).

We record a notation and an elementary observation needed in the final part of this section. For every $n \in \mathbb{N}$, we denote by $N C E(2 n)$ the collection of all the partitions $\pi \in N C(2 n)$ with the property that every block of $\pi$ has even cardinality. Observe that if $\pi \in N C E(2 n)$ and if $V=\left\{k_{1}<k_{2}<\cdots<k_{2 m}\right\}$ is a block of $\pi$, then the numbers $k_{1}, k_{2}, \ldots, k_{2 m}$ have alternating parities. Indeed, for every $i=1, \ldots, 2 m-1$, the set $\left\{k_{i}+1, k_{i}+2, \ldots, k_{i+1}-1\right\}$ is a union of blocks of $\pi$, and hence has even cardinality, which implies that $k_{i+1}$ is of opposite parity from $k_{i}$.

For a discussion of other elementary facts concerning $N C(n)$, we refer to [15, Lecture 9].

Remark 3.2 (Review of $R$-transforms). (1) The $R$-transform of a $*$-distribution $\mu \in \mathcal{D}_{\text {alg }}(1, *)$ is the series $R_{\mu} \in$ $\mathbb{C}_{0}\left\langle\left\langle z, z^{*}\right\rangle\right\rangle$, whose coefficients are uniquely determined by the requirement that they relate to the $*$-moments of $\mu$ by the formula

$$
\mathrm{Cf}_{w}\left(M_{\mu}\right)=\sum_{\pi \in N C(n)} \mathrm{Cf}_{w ; \pi}\left(R_{\mu}\right), \quad w \in \mathcal{W}^{+} \text {and } n=|w| .
$$

Equation (3.1) is the free probabilistic counterpart of (2.5). It is often referred to as the moment-cumulant formula for free cumulants (see [15, Lecture 11] for an explanation of this terminology).

One can also define the series $R_{\mu}$ by an equation involving the series $M_{\mu}$ and $R_{\mu}$ themselves (rather than their coefficients, as in (3.1)). More precisely, $R_{\mu}$ is the unique series in $\mathbb{C}_{0}\left\langle\left\langle z, z^{*}\right\rangle\right\rangle$ that satisfies the functional equation

$$
R_{\mu}\left(z\left(1+M_{\mu}\left(z, z^{*}\right)\right), z^{*}\left(1+M_{\mu}\left(z, z^{*}\right)\right)\right)=M_{\mu}\left(z, z^{*}\right)
$$


(see [15, Corollary 16.16]). This is the free probabilistic analogue of (2.2), but now we only have an implicit functional equation rather than an explicit formula describing the series $R_{\mu}$.

An easy inductive argument in the moment-cumulant formula (3.1) shows that one can recover $M_{\mu}$ from $R_{\mu}$ and that (as in Remark 2.4) we have a bijection

$$
\mathcal{D}_{\mathrm{alg}}(1, *) \ni \mu \mapsto R_{\mu} \in \mathbb{C}_{0}\left\langle\left\langle z, z^{*}\right\rangle\right\rangle .
$$

In other words, one can define a distribution $\mu \in \mathcal{D}_{\text {alg }}(1, *)$ by specifying its $R$-transform.

(2) Consider the framework of Remark 2.11, where we discussed the moment series and $\eta$-series $M_{a}, \eta_{a} \in \mathbb{C} \llbracket z \rrbracket$ associated to an element $a$ in a noncommutative probability space $(\mathcal{A}, \varphi)$. In that framework one also has an $R$ transform associated with the element $a \in \mathcal{A}$. This is the series $R_{a} \in \mathbb{C} \llbracket z \rrbracket$ that relates to $M_{a}$ by

$$
\mathrm{Cf}_{n}\left(M_{a}\right)=\sum_{\pi \in N C(n)} \mathrm{Cf}_{n ; \pi}\left(R_{a}\right), \quad n \in \mathbb{N}
$$

and

$$
R_{a}\left(z\left(1+M_{a}(z)\right)\right)=M_{a}(z) .
$$

These formulas are analogous to (3.1) or (3.2), respectively. For a detailed discussion of the algebraic aspects of $R$ transforms (covering both the series $R_{\mu}$ in part (1) of this remark and the series $R_{a}$ in part (2)), see [15, Lecture 16].

Definition 3.3. (1) A $*$-distribution $\mu \in \mathcal{D}_{\text {alg }}(1, *)$ is said to be $R$-diagonal when $\mathrm{Cf}_{w}\left(R_{\mu}\right)=0$ for every word $w \in \mathcal{W}^{+}$that is not alternating.

(2) If $\mu$ is $R$-diagonal, its $R$-transform is thus of the form

$$
R_{\mu}\left(z, z^{*}\right)=\sum_{n=1}^{\infty} \alpha_{n}\left(z z^{*}\right)^{n}+\sum_{n=1}^{\infty} \beta_{n}\left(z^{*} z\right)^{n},
$$

with $\alpha_{n}, \beta_{n} \in \mathbb{C}$ for $n \in \mathbb{N}$. The sequences $\left(\alpha_{n}\right)_{n=1}^{\infty}$ and $\left(\beta_{n}\right)_{n=1}^{\infty}$ are called the determining sequences of $\mu$.

Remark 3.4. The concept of an $\eta$-diagonal $*$-distribution from Section 2 obviously parallels the one of an $R$-diagonal $*$-distribution, with the $\eta$-series in place of the $R$-transform. The basic properties of $\eta$-diagonal distributions proved in Section 2 are the counterparts of known facts concerning $R$-diagonal distributions, as noted below.

(1) Remark 2.11 is the Boolean counterpart of [15, Corollary 15.11, p. 244]: if $\mu$ is $R$-diagonal, then $Z^{*} Z$ and $Z Z^{*}$ are freely independent in the noncommutative probability space $\left(\mathbb{C}\left\langle Z, Z^{*}\right\rangle, \mu\right)$.

(2) Theorem 2.8 is the counterpart of [13, Theorem 1.2.1] which describes $R$-diagonal distributions in terms of their $*$-moments.

(3) Proposition 2.13 is analogous to [15, Proposition 15.6, p. 241] which gives a precise formula, first found in [11] for the coefficients of the one-variable $R$-transforms $R_{Z Z *}$ and $R_{Z * Z}$ in terms of the determining sequences $\left(\alpha_{n}\right)_{n=1}^{\infty}$ and $\left(\beta_{n}\right)_{n=1}^{\infty}$ from (3.5). This formula is more elaborate than the relation found in Proposition 2.13 for $\eta$-diagonal elements. It states that

$$
\left\{\begin{array}{l}
\mathrm{Cf}_{n}\left(R_{Z Z^{*}}\right)=\sum_{\pi=\left\{V_{1}, \ldots, V_{k}\right\} \in N C(n)} \alpha_{\left|V_{1}\right|} \beta_{\left|V_{2}\right|} \cdots \beta_{\left|V_{k}\right|} \quad \text { and } \\
\mathrm{Cf}_{n}\left(R_{Z^{*} Z}\right)=\sum_{\pi=\left\{V_{1}, \ldots, V_{k}\right\} \in N C(n)} \beta_{\left|V_{1}\right|} \alpha_{\left|V_{2}\right|} \cdots \alpha_{\left|V_{k}\right|},
\end{array}\right.
$$

where the blocks of $\pi$ are arranged so that $1 \in V_{1}$. For example, the first three coefficients of $R_{Z Z *}$ are $\alpha_{1}, \alpha_{2}+\alpha_{1} \beta_{1}$ and $\alpha_{3}+2 \alpha_{2} \beta_{1}+\alpha_{1} \beta_{2}+\alpha_{1} \beta_{1}^{2}$.

Equations (3.6) lead to the following observation: an easy induction on $n$ (where one singles out the terms indexed by the partition $1_{n} \in N C(n)$ on the right-hand sides of the equations) shows that the determining sequences $\left(\alpha_{n}\right)_{n=1}^{\infty}$ and $\left(\beta_{n}\right)_{n=1}^{\infty}$ can be retrieved from the coefficients of $R_{Z Z^{*}}$ and $R_{Z^{*} Z}$. Hence the $R$-diagonal $*$-distribution $\mu \in$ $\mathcal{D}_{\text {alg }}(1, *)$ is completely determined by these $R$-transforms. 
In Section 6 we require a reformulation of (3.6) in terms of operations with series rather than individual coefficients. This reformulation is given in the next proposition. The formulas (3.7) bear a striking resemblance to the functional equation (3.4) of the $R$-transform. In fact, (3.7) collapse to $R_{Z Z^{*}}(z)=R_{Z^{*} Z}(z)=M_{a}(z)$ in the special case $\alpha_{n}=\beta_{n}$, $n \in \mathbb{N}$, in which case we can take $a=b$.

Proposition 3.5. Let $\mu \in \mathcal{D}_{\mathrm{alg}}(1, *)$ be an $R$-diagonal $*$-distribution with determining sequences $\left(\alpha_{n}\right)_{n=1}^{\infty}$ and $\left(\beta_{n}\right)_{n=1}^{\infty}$. Suppose we are given some elements $a$ and $b$ in a noncommutative probability space $(\mathcal{A}, \varphi)$ such that

$$
R_{a}(z)=\sum_{n=1}^{\infty} \alpha_{n} z^{n} \quad \text { and } \quad R_{b}(z)=\sum_{n=1}^{\infty} \beta_{n} z^{n}
$$

Then, in the noncommutative probability space $\left(\mathbb{C}\left\langle Z, Z^{*}\right\rangle, \mu\right)$, we have

$$
R_{Z Z^{*}}(z)=R_{a}\left(z\left(1+M_{b}(z)\right)\right) \quad \text { and } \quad R_{Z^{*} Z}(z)=R_{b}\left(z\left(1+M_{a}(z)\right)\right) .
$$

Proof. The argument is analogous to the proof of (3.2) (see, for instance, the proof of [15, Theorem 16.15]). For the reader's convenience, we describe the basic idea.

By symmetry, it suffices to prove the first equality in (3.7). We show that the coefficients of $z^{n}$ in the series $R_{Z Z^{*}}(z)$ and $R_{a}\left(z\left(1+M_{b}(z)\right)\right)$ are equal to each other for every $n \in \mathbb{N}$. The formal series expansion

$$
R_{a}\left(z\left(1+M_{b}(z)\right)\right)=\sum_{m=1}^{\infty} \alpha_{m}\left(z\left(1+M_{b}(z)\right)\right)^{m}
$$

yields

$$
\mathrm{Cf}_{n}\left(R_{a}\left(z\left(1+M_{b}(z)\right)\right)\right)=\sum_{m=1}^{n} \alpha_{m} \mathrm{Cf}_{n-m}\left(\left(1+M_{b}\right)^{m}\right), \quad n \in \mathbb{N} .
$$

Recall that the coefficients of $1+M_{b}$ are moments of $b$, and expand $\left(1+M_{b}\right)^{m}$, to obtain

$$
\mathrm{Cf}_{n}\left[R_{a}\left(z\left(1+M_{b}\right)(z)\right)\right]=\sum_{m=1}^{n} \sum_{\begin{array}{c}
k_{1}, \ldots, k_{m} \geq 0 \\
\text { with } k_{1}+\cdots+k_{m}=n-m
\end{array}} \alpha_{m} \varphi\left(b^{k_{1}}\right) \cdots \varphi\left(b^{k_{m}}\right) .
$$

On the other hand, (3.6) yields

$$
\mathrm{Cf}_{n}\left(R_{Z Z^{*}}\right)=\sum_{m=1}^{n} \sum_{\substack{S \subseteq\{1, \ldots, n\} \text { with } \pi=\left\{V_{1}, \ldots, V_{p}\right\} \in N C(n) \\ \text { with } V_{1}=S}} \alpha_{m} \beta_{\left|V_{2}\right|} \cdots \beta_{\left|V_{p}\right|}, \quad n \in \mathbb{N} \text { and } 1 \in S \text {. }
$$

For a fixed set $S=\left\{s_{1}, \ldots, s_{m}\right\} \subseteq\{1, \ldots, n\}$ with $1=s_{1}<s_{2}<\cdots<s_{m} \leq n$, the collection of non-crossing partitions $\{\pi \in N C(n): S$ is a block of $\pi\}$ is naturally identified with the Cartesian product

$$
N C\left(s_{2}-s_{1}-1\right) \times \cdots \times N C\left(s_{m}-s_{m-1}-1\right) \times N C\left(n-s_{m}\right),
$$

in a way that converts the sum

$$
\sum_{\substack{\pi=\left\{V_{1}, \ldots, V_{p}\right\} \in N C(n) \\ \text { with } V_{1}=S}} \beta_{\left|V_{2}\right|} \cdots \beta_{\left|V_{p}\right|}
$$


into the product

$$
\prod_{\ell=1}^{m}\left(\sum_{\pi_{\ell} \in N C\left(s_{\ell+1}-s_{\ell}-1\right)} \prod_{V \in \pi_{\ell}} \beta_{|V|}\right),
$$

where we set $s_{m+1}=n+1$. An application of the moment-cumulant formula (3.3) shows that $\prod_{V \in \pi_{\ell}} \beta_{|V|}=$ $\varphi\left(b^{s_{\ell+1}-s_{\ell}-1}\right)$. (See the proof of [15, Theorem 16.15] for more details.) We conclude that

$$
\mathrm{Cf}_{n}\left(R_{Z Z^{*}}\right)=\sum_{m=1}^{n} \sum_{\substack{S \subseteq\{1, \ldots, n\} \text { with } \\|S|=m}} \alpha_{m} \prod_{\ell=1}^{m} \varphi\left(b^{s_{\ell+1}-s_{\ell}-1}\right), \quad n \in \mathbb{N} .
$$

Finally, observe that for every fixed $m \in\{1, \ldots, n\}$ there is a natural bijection between tuples $\left(k_{1}, \ldots, k_{m}\right) \in(\mathbb{N} \cup\{0\})^{m}$ with $k_{1}+\cdots+k_{m}=n-m$ (on the one hand) and subsets $1 \in S \subseteq\{1, \ldots, n\}$ with $|S|=m$ (on the other), given by the formula

$$
\left(k_{1}, \ldots, k_{m}\right) \mapsto S=\left\{1, k_{1}+2, k_{1}+k_{2}+3, \ldots, k_{1}+\cdots+k_{m-1}+m\right\} .
$$

The inner sums on the right-hand sides of (3.8) and (3.10) are identified term by term via this bijection, and this concludes the proof.

In the remainder of this section we discuss the $R$-diagonal $*$-distributions that satisfy the KMS condition. This is a special case of the class of $*$-distribution studied in [16] (see, for instance [16, Remark 2.10]). The best known example of a KMS $R$-diagonal distribution is the one where, in the framework of the next definition, one sets $\alpha_{1}=\lambda$, $\beta_{1}=1$ and $\alpha_{n}=\beta_{n}=0$ for $n \geq 2$; this is called the $\lambda$-circular distribution, and is studied in detail in [16, Section 4].

Definition 3.6. Let $\mu \in \mathcal{D}_{\text {alg }}(1, *)$ be an $R$-diagonal $*$-distribution with determining sequences $\left(\alpha_{n}\right)_{n=1}^{\infty}$ and $\left(\beta_{n}\right)_{n=1}^{\infty}$, and let $t$ be a positive real number. We say that $\mu$ satisfies the KMS condition with parameter $t$ if

$$
\alpha_{n}=t \beta_{n}, \quad n \in \mathbb{N} \text {. }
$$

The following result shows that the KMS condition is a generalization of the trace property, where the latter property occurs for the value $t=1$ of the parameter.

Proposition 3.7. Let $\mu \in \mathcal{D}_{\mathrm{alg}}(1, *)$ be an $R$-diagonal $*$-distribution, let $t$ be a positive real number, and suppose that $\mu$ satisfies the KMS condition with parameter $t$. Denote by

$$
U_{t}: \mathbb{C}\left\langle Z, Z^{*}\right\rangle \rightarrow \mathbb{C}\left\langle Z, Z^{*}\right\rangle
$$

the unique unital algebra homomorphism such that

$$
U_{t}(Z)=t Z \quad \text { and } \quad U_{t}\left(Z^{*}\right)=\frac{1}{t} Z^{*}
$$

Then

$$
\mu(P Q)=\mu\left(Q U_{t}(P)\right), \quad P, Q \in \mathbb{C}\left\langle Z, Z^{*}\right\rangle .
$$

Proof. Both sides of (3.12) are bilinear in $P$ and $Q$, so it suffices to check the equation when both $P$ and $Q$ are monomials. Using the notation $Z^{\varnothing}=1$, we must show that

$$
\mu\left(Z^{v} Z^{w}\right)=\mu\left(Z^{w} U_{t}\left(Z^{v}\right)\right), \quad v, w \in \mathcal{W}^{+} \cup\{\varnothing\} .
$$


Equivalently, we must show that the set

$$
\mathcal{S}=\left\{v \in \mathcal{W}^{+} \cup\{\varnothing\}: \mu\left(Z^{v} Z^{w}\right)=\mu\left(Z^{w} U_{t}\left(Z^{v}\right)\right), w \in \mathcal{W}^{+} \cup\{\phi\}\right\}
$$

is equal to $\mathcal{W}^{+} \cup\{\varnothing\}$. The set $\mathcal{S}$ is clearly closed under concatenation and contains $\varnothing$. Therefore, it suffices to show that $\{1, *\} \subset \mathcal{S}$. In other words it suffices to prove that

$$
\mu\left(Z Z^{w}\right)=t \mu\left(Z^{w} Z\right) \quad \text { and } \quad \mu\left(Z^{*} Z^{w}\right)=\frac{1}{t} \mu\left(Z^{w} Z^{*}\right), \quad w \in \mathcal{W}^{+} \cup\{\varnothing\} .
$$

We only prove the first equality in (3.14); the verification of the second one is analogous. The case $w=\varnothing$ follows from the fact (incorporated in the definition of an $R$-diagonal distribution) that $\mu(Z)=0$. For the remainder part of the proof we fix a word $w=\left(\ell_{1}, \ldots, \ell_{n}\right) \in \mathcal{W}^{+}$, for which we prove that $\mu\left(Z Z^{w}\right)=t \mu\left(Z^{w} Z\right)$. Observe that

$$
\mu\left(Z Z_{w}\right)=\mathrm{Cf}_{w_{1}}\left(M_{\mu}\right), \quad \mu\left(Z_{w} Z\right)=\mathrm{Cf}_{w_{2}}\left(M_{\mu}\right),
$$

where $w_{1}:=\left(1, \ell_{1}, \ldots, \ell_{n}\right)$ and $w_{2}:=\left(\ell_{1}, \ldots, \ell_{n}, 1\right)$. It is convenient to view $w_{1}$ and $w_{2}$ as functions from $\{1, \ldots, n+1\}$ to $\{1, *\}$ and to record the fact that

$$
w_{2}=w_{1} \circ \gamma_{n+1}
$$

where $\gamma_{n+1}$ is the cyclic permutation $1 \mapsto 2 \mapsto \cdots \mapsto n+1 \mapsto 1$ of $\{1, \ldots, n+1\}$.

For every partition $\pi=\left\{V_{1}, \ldots, V_{k}\right\} \in N C(n+1)$ we denote by $\gamma_{n+1}^{-1}(\pi)$ the partition (still in $N C(n+1)$ ) whose blocks are $\gamma_{n+1}^{-1}\left(V_{1}\right), \ldots, \gamma_{n+1}^{-1}\left(V_{k}\right)$. The desired conclusion $\mathrm{Cf}_{w_{1}}\left(M_{\mu}\right)=t \mathrm{Cf}_{w_{2}}\left(M_{\mu}\right)$ is obtained from

$$
\mathrm{Cf}_{w_{1} ; \pi}\left(R_{\mu}\right)=t \mathrm{Cf}_{w_{2} ; \gamma_{n+1}^{-1}(\pi)}\left(R_{\mu}\right), \quad \pi \in N C(n+1),
$$

using the moment-cumulant formula. Indeed, sum both sides of (3.16) over $\pi \in N C(n+1)$ and invoke (3.1) applied to the words $w_{1}$ and $w_{2}$. The sums thus obtained are precisely $\mathrm{Cf}_{w_{1}}\left(M_{\mu}\right)$ on the left side and $t \mathrm{Cf}_{w_{2}}\left(M_{\mu}\right)$ on the right.

Thus, it remains to prove (3.16). Fix a partition $\pi=\left\{V_{1}, \ldots, V_{k}\right\} \in N C(n+1)$ such that $1 \in V_{1}$. Then $\gamma_{n+1}^{-1}(\pi)=$ $\left\{W_{1}, \ldots, W_{k}\right\}$, where $W_{j}=\gamma_{n+1}^{-1}\left(V_{j}\right), 1 \leq j \leq k$, and $n+1 \in W_{1}$. It follows from (3.15) that $w_{1}\left|V_{j}=w_{2}\right| W_{j} \in \mathcal{W}^{+}$ if $2 \leq j \leq k$, and thus

$$
\mathrm{Cf}_{w_{1} \mid V_{j}}\left(R_{\mu}\right)=\mathrm{Cf}_{w_{2} \mid W_{j}}\left(R_{\mu}\right), \quad 2 \leq j \leq k
$$

For the remaining block, we show that

$$
\mathrm{Cf}_{w_{1} \mid V_{1}}\left(R_{\mu}\right)=t \cdot \mathrm{Cf}_{w_{2} \mid W_{1}}\left(R_{\mu}\right) .
$$

Indeed, suppose that $V_{1}=\left\{j_{1}, \ldots, j_{m}\right\}$ with $1=j_{1}<j_{2}<\cdots<j_{m}$, and therefore $W_{1}=\left\{j_{2}-1, \ldots, j_{m}-1, n+1\right\}$. Both sides of (3.18) are 0 if $m$ is odd or if $m$ is even but $w_{1} \mid V_{1}$ is not an alternating word. If $m$ is even and $w_{1} \mid V_{1}$ is alternating, then we find that $w_{1} \mid V_{1}=\{1, *\}^{m / 2}$ and $w_{2} \mid V_{2}=\{*, 1\}^{m / 2}$, which implies that $\mathrm{Cf}_{w_{1} \mid V_{1}}\left(R_{\mu}\right)=\alpha_{m / 2}$ and $\mathrm{Cf}_{w_{2} \mid W_{1}}\left(R_{\mu}\right)=\beta_{m / 2}$. In this case, (3.18) follows from the KMS hypothesis.

Finally, for the partition $\pi$ fixed in the preceding paragraph we write:

$$
\begin{aligned}
\mathrm{Cf}_{w_{1} ; \pi}\left(R_{\mu}\right) & =\mathrm{Cf}_{w_{1} \mid V_{1}}\left(R_{\mu}\right) \prod_{j=2}^{k} \mathrm{Cf}_{w_{1} \mid V_{j}}\left(R_{\mu}\right) \\
& =t \mathrm{Cf}_{w_{2} \mid W_{1}}\left(R_{\mu}\right) \prod_{j=2}^{k} \mathrm{Cf}_{w_{2} \mid W_{j}}\left(R_{\mu}\right) \quad(\text { by (3.17) and (3.18)) } \\
& =t \mathrm{Cf}_{w_{2} ; \gamma_{n+1}^{-1}(\pi)}\left(R_{\mu}\right),
\end{aligned}
$$

thus concluding the proof of (3.16). 
Remark 3.8. The converse of Proposition 3.7 is also true. More precisely, every $R$-diagonal distribution $\mu \in$ $\mathcal{D}_{\text {alg }}(1, *)$ that satisfies (3.12) for some $t \in(0,+\infty)$ must also satisfy the KMS condition for the same value of $t$. To see this, let $\left(\alpha_{n}\right)_{n=1}^{\infty}$ and $\left(\beta_{n}\right)_{n=1}^{\infty}$ be the determining sequences of $\mu$. Equation (3.12) yields, in particular, the identity

$$
\mu\left(\left(Z Z^{*}\right)^{n}\right)=t \mu\left(\left(Z^{*} Z\right)^{n}\right), \quad n \in \mathbb{N} .
$$

This identity implies $\alpha_{n}=t \beta_{n}, n \in \mathbb{N}$, by induction on $n$. For the induction step one invokes the moment-cumulant formula in order to expand both sides of (3.19) as sums over $N C(2 n)$; then the action of the cyclic permutation $\gamma_{2 n}^{-1}$ on $N C(2 n)$ can be used in the same way as it was done in the proof of Proposition 3.7.

\section{The framework of $\mathcal{D}_{c}(1, *)$ and $\mathcal{D}_{c}(k)$, BBP bijections}

We now introduce the analytic framework which is of interest for the present paper.

Definition 4.1. (1) Let $(\mathcal{A}, \varphi)$ be a $C^{*}$-probability space (which means that $\mathcal{A}$ is a unital $C^{*}$-algebra, $\varphi: \mathcal{A} \rightarrow \mathbb{C}$ is a positive linear functional, and $\varphi(1)=1)$, and let $a \in \mathcal{A}$. The $*$-distribution of $a$ is the functional $\mu \in \mathcal{D}_{\text {alg }}(1, *)$ determined by the requirement that

$$
\mu\left(Z^{w}\right)=\varphi\left(a^{w}\right), \quad w \in \mathcal{W}^{+} .
$$

(2) We denote by $\mathcal{D}_{c}(1, *)$ the set of all elements of $\mathcal{D}_{\text {alg }}(1, *)$ that are equal to the $*$-distribution of some element in a $C^{*}$-probability space.

(3) Free additive (respectively, multiplicative) convolution is a binary operation on $\mathcal{D}_{c}(1, *)$ denoted by $\boxplus$ (respectively, $\otimes)$. This operation is uniquely determined by the following property: given elements $a, a^{\prime}$ in a $C^{*}$-probability space $(\mathcal{A}, \varphi)$ such that $\left\{a, a^{*}\right\}$ is free from $\left\{a^{\prime},\left(a^{\prime}\right)^{*}\right\}$, the $*$-distribution of $a+a^{\prime}$ (respectively, $\left.a a^{\prime}\right)$ is the free additive (respectively, multiplicative) convolution of the $*$-distributions of $a$ and $a^{\prime}$. See [15, Lectures 5 and 7] for more details. that

(4) An element $\mu \in \mathcal{D}_{c}(1, *)$ is said to be $\boxplus$-infinitely divisible if for every $n \in \mathbb{N}$ there exists $\mu_{n} \in \mathcal{D}_{c}(1, *)$ such

$$
\mu=\underbrace{\mu_{n} \boxplus \cdots \boxplus \mu_{n}}_{n \text { times }}
$$

The set of all $\boxplus$-infinitely divisible distributions in $\mathcal{D}_{c}(1, *)$ is denoted by $\mathcal{D}_{c}^{\text {(inf-div) }}(1, *)$.

The main result of this section is the following theorem. The series $R_{v}$ and $\eta_{\mu}$ appearing in the statement of the theorem are as defined in Sections 2 and 3.

Theorem 4.2 (BBP bijection on $\left.\mathcal{D}_{c}(1, *)\right)$. There exists a bijection

$$
\mathbb{B}_{(1, *)}: \mathcal{D}_{c}(1, *) \rightarrow \mathcal{D}_{c}^{\text {(inf-div) }}(1, *),
$$

determined by the requirement that

$$
R_{\mathbb{B}_{(1, *)}(\mu)}=\eta_{\mu}, \quad \mu \in \mathcal{D}_{c}(1, *) .
$$

More precisely, for every $\mu \in \mathcal{D}_{c}(1, *)$ there exists a unique $*$-distribution $v \in \mathcal{D}_{c}^{(\text {inf-div) }}(1, *)$ such that $R_{\nu}=\eta_{\mu}$, and we define $\mathbb{B}_{(1, *)}(\mu):=v$.

Definition and Remark 4.3 (Framework of $\mathcal{D}_{c}(k)$ ). We reduce Theorem 4.2 to an analogous theorem proved in [3] for the space, denoted by $\mathcal{D}_{c}(2)$, of joint distributions of pairs of selfadjoint elements in a $C^{*}$-probability space. The passage from $\mathcal{D}_{c}(1, *)$ to $\mathcal{D}_{c}(2)$ is natural, and essentially amounts to the change of variables

$$
\left(a, a^{*}\right) \mapsto\left(\frac{a+a^{*}}{2}, \frac{a-a^{*}}{2 i}\right),
$$


for $a$ in a $C^{*}$-probability space $(\mathcal{A}, \varphi)$. In order to clarify this idea, we review briefly the framework of $\mathcal{D}_{c}(k)$. Fix $k \in \mathbb{N}$.

(1) We denote by $\mathbb{C}\left\langle X_{1}, \ldots, X_{k}\right\rangle$ the algebra of polynomials in the non-commuting indeterminates $X_{1}, \ldots, X_{k}$.

(2) Let $(\mathcal{A}, \varphi)$ be a $C^{*}$-probability space and let $b_{1}, \ldots, b_{k} \in \mathcal{A}$ be selfadjoint. The joint distribution of $b_{1}, \ldots, b_{k}$ is the linear functional $\lambda: \mathbb{C}\left\langle X_{1}, \ldots, X_{k}\right\rangle \rightarrow \mathbb{C}$ which is determined by the requirement that $\lambda(1)=1$ and

$$
\lambda\left(X_{i_{1}} \cdots X_{i_{n}}\right)=\varphi\left(b_{i_{1}} \cdots b_{i_{n}}\right), \quad n \in \mathbb{N}, i_{1}, \ldots, i_{n} \in\{1, \ldots, k\} .
$$

(3) We denote by $\mathcal{D}_{c}(k)$ the set of all linear functionals $\lambda: \mathbb{C}\left\langle X_{1}, \ldots, X_{k}\right\rangle \rightarrow \mathbb{C}$ that can arise as joint distributions of $k$-tuples of selfadjoint elements in some $C^{*}$-probability space.

(4) Free additive convolution is a binary operation on $\mathcal{D}_{c}(k)$ denoted ${ }^{4}$ by $\boxplus$. This operation is uniquely determined by the following property: given selfadjoint elements $a_{1}, \ldots, a_{k}$ and $b_{1}, \ldots, b_{k}$ in a $C^{*}$-probability space $(\mathcal{A}, \varphi)$ such that $\left\{a_{1}, \ldots, a_{k}\right\}$ is free from $\left\{b_{1}, \ldots, b_{k}\right\}$, the joint distribution of $a_{1}+b_{1}, \ldots, a_{k}+b_{k}$ is the free additive convolution of the joint distributions of $a_{1}, \ldots, a_{k}$ and $b_{1}, \ldots, b_{k}$. The concept of $\boxplus$-infinite divisibility in $\mathcal{D}_{c}(k)$ is introduced as in Definition 4.1(4). The set of $\boxplus$-infinitely divisible distributions in $\mathcal{D}_{c}(k)$ is denoted by $\mathcal{D}_{c}^{\text {(inf-div) }}(k)$.

(5) We denote by $\mathbb{C}_{0}\left\langle\left\langle x_{1}, \ldots, x_{k}\right\rangle\right\rangle$ the space of those formal power series with complex coefficients in $k$ noncommuting indeterminates $x_{1}, \ldots, x_{k}$ whose constant term is equal to 0 . We denote by $\operatorname{Cf}_{\left(i_{1}, \ldots, i_{n}\right)}(f)$ the coefficient of $x_{i_{1}} \cdots x_{i_{n}}$ in a series $f \in \mathbb{C}_{0}\left\langle\left\langle x_{1}, \ldots, x_{k}\right\rangle\right\rangle$. Every joint distribution $\lambda \in \mathcal{D}_{c}(k)$ has a moment series $M_{\lambda}$, an $R$-transform $R_{\lambda}$ and an $\eta$-series $\eta_{\lambda}$. These are elements of $\mathbb{C}_{0}\left\langle\left\langle x_{1}, \ldots, x_{k}\right\rangle\right\rangle$, and their definitions are analogous to Definitions 2.3 and 3.2. A detailed description of these power series and of the relations between their coefficients can be found in [3, pp. 14-17].

The proof of Theorem 4.2 will be reduced to the following result from [3] (see also [5] for the case $k=1$ ).

Theorem 4.4 (BBP bijection on $\left.\mathcal{D}_{c}(k)\right)$. Let $k$ be a positive integer. There exists a bijection $\mathbb{B}_{k}: \mathcal{D}_{c}(k) \rightarrow$ $\mathcal{D}_{c}^{\text {(inf-div) }}(k)$, determined by the requirement that

$$
R_{\mathbb{B}_{k}(\lambda)}=\eta_{\lambda}, \quad \lambda \in \mathcal{D}_{c}(k)
$$

More precisely, for every $\lambda \in \mathcal{D}_{c}(k)$ there exists a unique $v \in \mathcal{D}_{c}^{\text {(inf-div) }}(k)$ such that $R_{v}=\eta_{\lambda}$, and we define $\mathbb{B}_{k}(\lambda):=v$.

Remark 4.5. We only need Theorem 4.4 for $k=1$ and $k=2$. When $k=1$, the space $\mathcal{D}_{c}(1)$ is naturally identified with the space $\mathcal{P}_{c}$ of compactly supported Borel probability measures on $\mathbb{R}$. Indeed, given $b=b^{*}$ in a $C^{*}$-probability space $(\mathcal{A}, \varphi)$, Definition 4.3(2) produces a linear functional $\lambda: \mathbb{C}\left\langle x_{1}\right\rangle \rightarrow \mathbb{C}$ which becomes, via the Riesz representation theorem, a Borel probability measure supported on the spectrum of $b$. The original BBP bijection from [5] was defined on $\mathcal{P}_{c}$ (and on the larger set $\mathcal{P}$ of all Borel probability measures on $\mathbb{R}$ ). In Section 6, we will simply talk about $\mathbb{B}(\sigma)$ for $\sigma \in \mathcal{P}_{c}$. In other words if $\lambda$ denotes the functional in $\mathcal{D}_{c}(1)$ corresponding to $\sigma$, then $\mathbb{B}(\sigma) \in \mathcal{P}_{c}$ denotes the probability measure corresponding to $\mathbb{B}_{1}(\lambda)$.

The following result creates bijections $C$ and $D$ that we use in conjunction with the case $k=2$ of Theorem 4.4. (The letters $C$ and $D$ are meant to suggest complexification and decomplexification.) The proof is immediate, and therefore omitted.

Proposition 4.6. There exists a bijection $D: \mathcal{D}_{c}(1, *) \rightarrow \mathcal{D}_{c}(2)$ defined as follows. Given $\mu \in \mathcal{D}_{c}(1, *)$ that is the *-distribution of an element a in a $C^{*}$-probability space $(\mathcal{A}, \varphi), D(\mu)$ is the joint distribution of the pair

$$
\left(\frac{a+a^{*}}{2}, \frac{a-a^{*}}{2 i}\right)
$$

The inverse of $D$ is the bijection $C: \mathcal{D}_{c}(2) \rightarrow \mathcal{D}_{c}(1, *)$ defined as follows. Given $\lambda \in \mathcal{D}_{c}(2)$ that is the joint distribution of a pair $b_{1}, b_{2}$ of selfadjoint elements in a $C^{*}$-probability space $(\mathcal{A}, \varphi), C(\lambda)$ is the $*$-distribution of $b_{1}+i b_{2}$.

\footnotetext{
${ }^{4}$ It is customary to always denote free additive convolution by “ $\boxplus$ ”. The setting in which the symbol $\boxplus$ is used should be clear, in each case, from the context.
} 
Definition and Remark 4.7. In addition to the transformations $C$ and $D$, the proof of Theorem 4.2 requires the corresponding change of variables for power series. Denote by

$$
t_{1,1}=t_{1, *}=\frac{1}{2} \quad \text { and } \quad t_{2,1}=\frac{1}{2 i}, \quad t_{2, *}=-\frac{1}{2 i},
$$

the coefficients of the linear transformation $b_{1}=\left(a+a^{*}\right) / 2, b_{2}=\left(a-a^{*}\right) / 2 i$. This transformation can now be written more compactly as

$$
b_{i}=\sum_{\ell \in\{1, *\}} t_{i, \ell} a^{\ell}, \quad \text { for } i=1,2 .
$$

Using the coefficients $t_{i, \ell}$ we define a map $\widetilde{D}: \mathbb{C}_{0}\left\langle\left\langle z, z^{*}\right\rangle\right\rangle \rightarrow \mathbb{C}_{0}\left\langle\left\langle x_{1}, x_{2}\right\rangle\right\rangle$ as follows: given a series $f \in \mathbb{C}_{0}\left\langle\left\langle z, z^{*}\right\rangle\right\rangle$, the coefficients of the series $g=\widetilde{D}(f) \in \mathbb{C}_{0}\left\langle\left\langle x_{1}, x_{2}\right\rangle\right\rangle$ are given by

$$
\mathrm{Cf}_{\left(i_{1}, \ldots, i_{n}\right)}(g):=\sum_{\ell_{1}, \ldots, \ell_{n} \in\{1, *\}} t_{i_{1}, \ell_{1}} \cdots t_{i_{n}, \ell_{n}} \mathrm{Cf}_{\left(\ell_{1}, \ldots, \ell_{n}\right)}(f), \quad n \in \mathbb{N}, i_{1}, \ldots, i_{n} \in\{1,2\} .
$$

The map $\widetilde{D}$ is clearly linear and bijective. Its inverse $\widetilde{C}: \mathbb{C}_{0}\left\langle\left\langle x_{1}, x_{2}\right\rangle\right\rangle \rightarrow \mathbb{C}_{0}\left\langle\left\langle z, z^{*}\right\rangle\right\rangle$ is defined by a formula analogous to (4.5), but with $\left[t_{i, \ell}\right]$ replaced by the inverse matrix

$$
t_{1,1}^{\prime}=1, \quad t_{1,2}^{\prime}=i \quad \text { and } \quad t_{*, 1}^{\prime}=1, \quad t_{*, 2}^{\prime}=-i
$$

Lemma 4.8. For every $*$-distribution $\mu \in \mathcal{D}_{c}(1, *)$, we have

$$
M_{D(\mu)}=\widetilde{D}\left(M_{\mu}\right), \quad R_{D(\mu)}=\widetilde{D}\left(R_{\mu}\right) \quad \text { and } \quad \eta_{D(\mu)}=\widetilde{D}\left(\eta_{\mu}\right) .
$$

Proof. Suppose that $\mu \in \mathcal{D}_{c}(1, *)$ is the $*$-distribution of an element $a$ in a $C^{*}$-probability space $(\mathcal{A}, \varphi)$, and set $b_{i}=\sum_{\ell \in\{1, *\}} t_{i, \ell} a^{\ell}$ for $i=1,2$. By definition, the joint distribution of $b_{1}, b_{2}$ is $D(\mu) \in \mathcal{D}_{c}(2)$. To verify the first identity in (4.6), fix $n \in \mathbb{N}$ and $i_{1}, \ldots, i_{n} \in\{1,2\}$, and calculate directly

$$
\begin{aligned}
\mathrm{Cf}_{\left(i_{1}, \ldots, i_{n}\right)}\left(M_{D(\mu)}\right) & =\varphi\left(b_{i_{1}} \cdots b_{i_{n}}\right) \\
& =\varphi\left(\left(t_{i_{1}, 1} a^{1}+t_{i_{1}, *} a^{*}\right) \cdots\left(t_{i_{n}, 1} a^{1}+t_{i_{n}, *} a^{*}\right)\right) \\
& =\sum_{\ell_{1}, \ldots, \ell_{n} \in\{1, *\}} t_{i_{1}, \ell_{1}} \cdots t_{i_{n}, \ell_{n}} \varphi\left(a^{\ell_{1}} \cdots a^{\ell_{n}}\right) \\
& =\sum_{\ell_{1}, \ldots, \ell_{n} \in\{1, *\}} t_{i_{1}, \ell_{1}} \cdots t_{i_{n}, \ell_{n}} \operatorname{Cf}_{\left(\ell_{1}, \ldots, \ell_{n}\right)}\left(M_{\mu}\right) \\
& =\mathrm{Cf}_{\left(i_{1}, \ldots, i_{n}\right)} \widetilde{D}\left(M_{\mu}\right) .
\end{aligned}
$$

The second equality in (4.6) follows from a similar multilinearity argument, using the fact that the $C^{*}$-probability space $(\mathcal{A}, \varphi)$ carries a family of multilinear functionals $\left(\kappa_{n}: \mathcal{A}^{n} \rightarrow \mathbb{C}\right)_{n=1}^{\infty}$, called free cumulant functionals, such that

$$
\begin{aligned}
& \mathrm{Cf}_{\left(i_{1}, \ldots, i_{n}\right)}\left(R_{D(\mu)}\right)=\kappa_{n}\left(b_{i_{1}}, \ldots, b_{i_{n}}\right), \quad n \in \mathbb{N}, i_{1}, \ldots, i_{n} \in\{1,2\} \quad \text { and } \\
& \operatorname{Cf}_{\left(\ell_{1}, \ldots, \ell_{n}\right)}\left(R_{\mu}\right)=\kappa_{n}\left(a^{\ell_{1}}, \ldots, a^{\ell_{n}}\right), \quad n \in \mathbb{N}, \ell_{1}, \ldots, \ell_{n} \in\{1, *\} .
\end{aligned}
$$

(This multilinearity argument is precisely the one used to describe the behavior of the $R$-transform under linear transformations [15, Proposition 16.12].)

The third equality (4.6) follows from a similar multilinearity argument, using the Boolean cumulant functionals $\left(\beta_{n}: \mathcal{A}^{n} \rightarrow \mathbb{C}\right)_{n=1}^{\infty}$ (for a discussion of Boolean cumulants see, for instance, [12, Section 4.6]).

Lemma 4.9. Let $D: \mathcal{D}_{c}(1, *) \rightarrow \mathcal{D}_{c}(2)$ be the bijection defined in Proposition 4.6. Then: 
(1) $D\left(\mu \boxplus \mu^{\prime}\right)=D(\mu) \boxplus D\left(\mu^{\prime}\right)$ for every $\mu, \mu^{\prime} \in \mathcal{D}_{c}(1, *)$.

(2) $D\left(\mathcal{D}_{c}^{\text {(inf-div) }}(1, *)\right)=\mathcal{D}_{c}^{\text {(inf-div) }}(2)$.

Proof. (1) Since every $\lambda \in \mathcal{D}_{c}(2)$ is uniquely determined by its $R$-transform, it suffices to verify that $D\left(\mu \boxplus \mu^{\prime}\right)$ and $D(\mu) \boxplus D\left(\mu^{\prime}\right)$ have the same $R$-transform. Indeed,

$$
\begin{aligned}
R_{D\left(\mu \boxplus \mu^{\prime}\right)} & =\widetilde{D}\left(R_{\mu \boxplus \mu^{\prime}}\right) \quad \text { (by Lemma 4.8) } \\
& =\widetilde{D}\left(R_{\mu}\right)+\widetilde{D}\left(R_{\mu^{\prime}}\right) \quad \text { (since } R_{\mu \boxplus \mu^{\prime}}=R_{\mu}+R_{\mu^{\prime}} \text { and } \widetilde{D} \text { is linear) } \\
& =R_{D(\mu)}+R_{D\left(\mu^{\prime}\right)} \quad \text { (by Lemma 4.8) } \\
& =R_{D(\mu) \boxplus D\left(\mu^{\prime}\right) .}
\end{aligned}
$$

Part (2) follows immediately from (1) and from the definition of $\boxplus$-infinite divisibility.

Proof of Theorem 4.2. We define the required bijection $\mathbb{B}_{(1, *)}$ so that the diagram

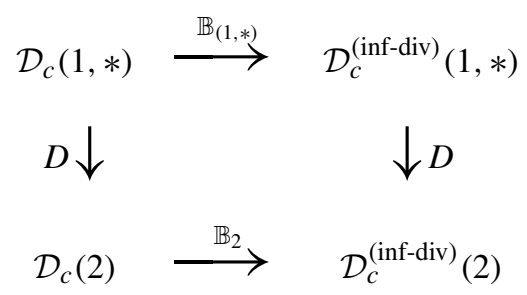

is commutative, where $D$ is defined in Proposition 4.6 and $\mathbb{B}_{2}$ is provided by Theorem 4.4 for $k=2$. More precisely, let $D_{0}: \mathcal{D}_{c}^{\text {(inf-div) }}(1, *) \rightarrow \mathcal{D}_{c}^{\text {(inf-div) }}(2)$ be the restriction of $D$; this is a bijection by Lemma 4.9(2). Then define

$$
\mathbb{B}_{(1, *)}:=D_{0}^{-1} \circ \mathbb{B}_{2} \circ D \text {. }
$$

Pick an arbitrary $\mu \in \mathcal{D}_{c}(1, *)$, denote $\mathbb{B}_{(1, *)}(\mu)=v$. We prove that $R_{v}=\eta_{\mu}$. Since $\widetilde{D}$ is injective, it suffices to verify that $\widetilde{D}\left(R_{\nu}\right)=\widetilde{D}\left(\eta_{\mu}\right)$. Indeed, the definition of $\mathbb{B}_{(1, *)}$ implies $\mathbb{B}_{2}(D(\mu))=D(v)$, and the definition of $\mathbb{B}_{2}$ yields $R_{D(v)}=\eta_{D(\mu)}$. Thus

$$
\widetilde{D}\left(R_{v}\right)=R_{D(v)}=\eta_{D(\mu)}=\widetilde{D}\left(\eta_{\mu}\right)
$$

as required.

\section{Parametrization of $\eta$-diagonal distributions in $\mathcal{D}_{c}(1, *)$}

We show that an $\eta$-diagonal $*$-distribution $\mu \in \mathcal{D}_{c}(1, *)$ is naturally parametrized by the pair of compactly supported probability measures on $[0, \infty)$ that arise as the distributions of $Z Z^{*}$ and of $Z^{*} Z$ in the noncommutative probability space $\left(\mathbb{C}\left\langle Z, Z^{*}\right\rangle, \mu\right)$.

Definition and Remark 5.1. Suppose that $\mu \in \mathcal{D}_{c}(1, *)$ is the $*$-distribution of an element $a$ in a $C^{*}$-probability space $(\mathcal{A}, \varphi)$. Basic considerations on positive elements in a $C^{*}$-probability space (see, for instance, [15, Propositions 3.13 and 3.6]) show the existence of compactly supported Borel probability measures $\sigma_{1}, \sigma_{2}$ on $[0, \infty)$ such that

$$
\varphi\left(\left(a a^{*}\right)^{n}\right)=\int_{0}^{\infty} t^{n} d \sigma_{1}(t) \quad \text { and } \varphi\left(\left(a^{*} a\right)^{n}\right)=\int_{0}^{\infty} t^{n} d \sigma_{2}(t), \quad n \in \mathbb{N} .
$$

Thus $\sigma_{1}$ and $\sigma_{2}$ satisfy

$$
\int_{0}^{\infty} t^{n} d \sigma_{1}(t)=\mu\left(\left(Z Z^{*}\right)^{n}\right), \quad n \in \mathbb{N}
$$


and

$$
\int_{0}^{\infty} t^{n} d \sigma_{2}(t)=\mu\left(\left(Z^{*} Z\right)^{n}\right), \quad n \in \mathbb{N} .
$$

Moreover, $\sigma_{1}$ and $\sigma_{2}$ are uniquely determined by (5.1) and (5.2) since a compactly supported probability measure on $\mathbb{R}$ is determined by its moments. We refer to $\sigma_{1}$ and $\sigma_{2}$ as the distributions of $Z Z^{*}$ and $Z^{*} Z$, respectively, in the *-probability space $\left(\mathbb{C}\left\langle Z, Z^{*}\right\rangle, \mu\right)$.

The following theorem provides the parametrization announced in the title of the section.

Theorem 5.2. Let $\mathcal{P}_{c}^{+}$denote the set of all compactly supported Borel probability measures on $[0, \infty)$. There is bijective map

$$
\Phi: \mathcal{P}_{c}^{+} \times \mathcal{P}_{c}^{+} \rightarrow\left\{\mu \in \mathcal{D}_{c}(1, *): \mu \text { is } \eta \text {-diagonal }\right\}
$$

described as follows: given $\sigma_{1}, \sigma_{2} \in \mathcal{P}_{c}^{+}, \Phi\left(\sigma_{1}, \sigma_{2}\right)$ is the unique $\eta$-diagonal $*$-distribution $\mu \in \mathcal{D}_{c}(1, *)$ such that the distributions of $Z Z^{*}$ and $Z^{*} Z$ in $\left(\mathbb{C}\left\langle Z, Z^{*}\right\rangle, \mu\right)$ are equal to $\sigma_{1}$ and $\sigma_{2}$, respectively.

The point of Theorem 5.2 is that the map $\Phi$ is defined on all of $\mathcal{P}_{c}^{+} \times \mathcal{P}_{c}^{+}$. In other words, for every $\sigma_{1}, \sigma_{2} \in \mathcal{P}_{c}^{+}$ there exists an $\eta$-diagonal $*$-distribution $\mu \in \mathcal{D}_{c}(1, *)$ such that (5.1) and (5.2) hold. We prove this by producing an operator model for $\mu$ : starting from $\sigma_{1}$ and $\sigma_{2}$ we construct explicitly an operator $A$ on a Hilbert space $\mathcal{K}$ such that the $*$-distribution of $A$ with respect to a suitably chosen functional on $B(\mathcal{K})$ is the required $\eta$-diagonal distribution. The bulk of this section is devoted to the description of the operator model. At the end, we complete the proof of Theorem 5.2. The construction of the operator model is described in the next remark.

Remark and Notation 5.3 (Description of the operator model). Fix $\sigma_{1}, \sigma_{2} \in \mathcal{P}_{c}^{+}$which we take as the input for our construction of an $\eta$-diagonal operator. In the description of the construction, it is convenient to use the symmetric square roots of $\sigma_{1}$ and $\sigma_{2}$. These are the symmetric compactly supported Borel probability measures $\widetilde{\sigma}_{1}$ and $\widetilde{\sigma}_{2}$ on $\mathbb{R}$ with moments given by the formula

$$
\int_{-\infty}^{\infty} t^{n} d \widetilde{\sigma}_{j}(t)= \begin{cases}0, & n \text { odd } \\ \int_{0}^{\infty} t^{n / 2} d \sigma_{j}(t), & n \text { even }\end{cases}
$$

for $j=1,2$. Our construction of an $\eta$-diagonal operator proceeds in three steps.

Step 1 . We construct a Hilbert space $\mathcal{H}$, a selfadjoint operator $X \in B(\mathcal{H})$, and vectors $\xi_{1}, \xi_{2} \in \mathcal{H}$ with the following properties:

(1a) $\left\|\xi_{1}\right\|=\left\|\xi_{2}\right\|=1$,

(1b) $\left\langle\xi_{1}, \xi_{2}\right\rangle=0$,

(1c) $\left\langle X^{k} \xi_{1}, \xi_{2}\right\rangle=\left\langle X^{k} \xi_{2}, \xi_{1}\right\rangle=0$ for $k \in \mathbb{N}$,

(1d) $\left\langle X^{2 k-1} \xi_{j}, \xi_{j}\right\rangle=0$ and $\left\langle X^{2 k} \xi_{j}, \xi_{j}\right\rangle=\int_{0}^{\infty} t^{k} d \sigma_{j}(t)$ for $k \in \mathbb{N}$ and $j=1,2$.

In other words, property (1d) says that $X$ has distribution $\widetilde{\sigma}_{1}$ with respect to the vector state defined by $\xi_{1}$, and distribution $\widetilde{\sigma}_{2}$ with respect to the one defined by $\xi_{2}$, on the operator algebra $B(\mathcal{H})$. For the actual construction of $X$ consider, for $j=1,2$, Hilbert spaces $\mathcal{M}_{j}$, selfadjoint operators $T_{j} \in B\left(\mathcal{M}_{j}\right)$, and unit vectors $\eta_{j} \in \mathcal{M}_{j}$, such that the distribution of $T_{j}$ with respect to the vector state $\eta_{j}$ is $\widetilde{\sigma}_{j}$. Then set $\mathcal{H}=\mathcal{M}_{1} \oplus \mathcal{M}_{2}, X=T_{1} \oplus T_{2}, \xi_{1}=\eta_{1} \oplus 0$, and $\xi_{2}=0 \oplus \eta_{2}$. Properties (1a)-(1d) are then easily verified. (In subsequent steps we only use the properties (1a)-(1d). The precise description of $\mathcal{H}, X, \xi_{1}$, and $\xi_{2}$ is not necessary.)

Step 2. Define a rank-one partial isometry $Y \in B(\mathcal{H})$ by setting $Y(\zeta)=\left\langle\zeta, \xi_{1}\right\rangle \xi_{2}$ for $\zeta \in \mathcal{H}$. We have $Y \xi_{1}=\xi_{2}$, $Y^{*} \zeta=\left\langle\zeta, \xi_{2}\right\rangle \xi_{1}$ for $\zeta \in \mathcal{H}$, and

$$
Y Y^{*} \zeta=\left\langle\zeta, \xi_{2}\right\rangle \xi_{2}, \quad Y^{*} Y \zeta=\left\langle\zeta, \xi_{1}\right\rangle \xi_{1} .
$$

Thus $Y Y^{*}$ and $Y^{*} Y$ are the orthogonal projections onto the 1-dimensional spaces generated by $\xi_{2}$ and $\xi_{1}$, respectively. 
Step 3. Consider the Hilbert space $\mathcal{K}=\mathcal{H} \otimes \mathcal{H}$ and the unit vector $\xi=\xi_{1} \otimes \xi_{2} \in \mathcal{K}$, then consider the $C^{*}$-probability space $\left(B(\mathcal{K}), \varphi_{\xi}\right)$, where $\varphi_{\xi}(T)=\langle T \xi, \xi\rangle$ for $T \in B(\mathcal{K})$. Let $V \in B(\mathcal{H} \otimes \mathcal{H})$ be the flip operator determined by the requirement that by $V\left(\zeta \otimes \zeta^{\prime}\right)=\zeta^{\prime} \otimes \zeta, \zeta, \zeta^{\prime} \in \mathcal{H}$. Note that $V$ is a symmetry (that is, it is self-adjoint and $V^{2}=I$ ). Finally, define $A=V(Y \otimes X)$.

This concludes the construction of the variable $A$ in $\left(B(\mathcal{K}), \varphi_{\xi}\right)$.

We now take on the proof that the operator $A$ constructed above has the desired $\eta$-diagonal distribution with respect to the functional $\varphi_{\xi}$. We start by recording some easily verified identities satisfied by $A$, the proof of which is left to the reader.

Lemma 5.4. Consider the framework of Remark 5.3. We have

$$
A A^{*}=X^{2} \otimes Y Y^{*}, \quad A^{*} A=Y^{*} Y \otimes X^{2}
$$

and

$$
A^{2}=X Y \otimes Y X, \quad\left(A^{*}\right)^{2}=Y^{*} X \otimes X Y^{*}
$$

The following lemma establishes the distributions of $A A^{*}$ and $A^{*} A$ along with a few non-alternating $*$-moments of $A$.

Lemma 5.5. Let $A$ and $\xi$ be as above, then for any integer $k \geq 0$ we have

(1) $\varphi_{\xi}\left(\left(A A^{*}\right)^{k}\right)=\int_{0}^{\infty} t^{k} d \sigma_{1}(t)$,

(2) $\varphi_{\xi}\left(A\left(A A^{*}\right)^{k}\right)=0$,

(3) $\varphi_{\xi}\left(A^{*}\left(A A^{*}\right)^{k}\right)=0$,

(4) $\varphi_{\xi}\left(\left(A^{*} A\right)^{k}\right)=\int_{0}^{\infty} t^{k} d \sigma_{2}(t)$,

(5) $\varphi_{\xi}\left(A\left(A^{*} A\right)^{k}\right)=0$,

(6) $\varphi_{\xi}\left(A^{*}\left(A^{*} A\right)^{k}\right)=0$.

Proof. We verify only the first three equations. The proof of (4)-(6) is similar. We have

$$
\left(A A^{*}\right)^{k}=X^{2 k} \otimes\left(Y Y^{*}\right)^{k}=X^{2 k} \otimes\left(Y Y^{*}\right),
$$

so

$$
\varphi_{\xi}\left(\left(A A^{*}\right)^{k}\right)=\left\langle\left(X^{2 k} \otimes\left(Y Y^{*}\right)\right) \xi, \xi\right\rangle=\left\langle X^{2 k} \xi_{1}, \xi_{1}\right\rangle,
$$

and (1) follows from property (1d) in Step 1 of the construction of $A$. To prove (2), we calculate

$$
A\left(A A^{*}\right)^{k}=V(Y \otimes X)\left(X^{2 k} \otimes Y Y^{*}\right),
$$

thus

$$
\begin{aligned}
\varphi_{\xi}\left(A\left(A A^{*}\right)^{k}\right) & =\left\langle(Y \otimes X)\left(X^{2 k} \otimes Y Y^{*}\right) \xi_{1} \otimes \xi_{2}, V \xi_{1} \otimes \xi_{2}\right\rangle \\
& =\left\langle(Y \otimes X)\left(X^{2 k} \xi_{1} \otimes \xi_{2}\right), \xi_{2} \otimes \xi_{1}\right\rangle \\
& =\left\langle Y X^{2 k} \xi_{1}, \xi_{2}\right\rangle\left\langle X \xi_{2}, \xi_{1}\right\rangle=0,
\end{aligned}
$$

because $\left\langle X \xi_{2}, \xi_{1}\right\rangle=0$, thereby concluding the proof of (2). Similarly,

$$
A^{*}\left(A A^{*}\right)^{k}=\left(X \otimes Y^{*}\right) V\left(X^{2 k} \otimes Y Y^{*}\right),
$$


SO

$$
\begin{aligned}
\varphi_{\xi}\left(A^{*}\left(A A^{*}\right)^{k}\right) & =\left\langle\left(X \otimes Y^{*}\right) V\left(X^{2 k} \otimes Y Y^{*}\right) \xi, \xi\right\rangle=\left\langle X \otimes Y^{*}\left(\xi_{2} \otimes X^{2 k} \xi_{1}\right), \xi_{1} \otimes \xi_{2}\right\rangle \\
& =\left\langle X \xi_{2}, \xi_{1}\right\rangle\left\langle Y^{*} X^{2 k} \xi_{1}, \xi_{2}\right\rangle=0
\end{aligned}
$$

by property (1c) in Step 1 of the construction of $A$.

The next lemma gives some properties of the operator $A$ that are useful in verifying its $\eta$-diagonality.

Lemma 5.6. Let $A$ and $\xi$ be as above, then for any integer $k \geq 0$ we have

(1) $A^{2}\left(A A^{*}\right)^{k} \xi=0$,

(2) $\left(A^{*}\right)^{2}\left(A A^{*}\right)^{k} \xi=0$,

(3) $\left(A^{*} A\right)\left(A A^{*}\right)^{k} \xi=\int_{0}^{\infty} t^{k} d \sigma_{1}(t) \cdot A^{*} A \xi$,

(4) $A^{2}\left(A^{*} A\right)^{k} \xi=0$,

(5) $\left(A^{*}\right)^{2}\left(A^{*} A\right)^{k} \xi_{=} 0$

(6) $\left(A A^{*}\right)\left(A^{*} A\right)^{k} \xi=\int_{0}^{\infty} t^{k} d \sigma_{2}(t) \cdot A A^{*} \xi$.

Proof. As in the previous proof we only verify (1)-(3). We have

$$
(A)^{2}\left(A A^{*}\right)^{k}=(X Y \otimes Y X)\left(X^{2 k} \otimes Y Y^{*}\right)=X Y X^{2 k} \otimes Y X Y Y^{*},
$$

and using the fact that $Y Y^{*} \xi_{2}=\xi_{2}$ we see that $Y X Y Y^{*} \xi_{2}=\left\langle X \xi_{2}, \xi_{1}\right\rangle \xi_{2}=0$ by property (1c). Similarly,

$$
\left(A^{*}\right)^{2}\left(A A^{*}\right)^{k}=\left(Y^{*} X \otimes X Y^{*}\right)\left(X^{2 k} \otimes Y Y^{*}\right)=Y^{*} X^{2 k+1} \otimes X Y^{*},
$$

and (2) follows because $Y^{*} X^{2 k+1} \xi_{1}=\left\langle X^{2 k+1} \xi_{1}, \xi_{2}\right\rangle \xi_{1}=0$ by (1c). Finally, (5.4) yields

$$
\left(A^{*} A\right)\left(A A^{*}\right)^{k}=\left(Y^{*} Y \otimes X^{2}\right)\left(X^{2 k} \otimes Y Y^{*}\right)=Y^{*} Y X^{2 k} \otimes X^{2} Y Y^{*} .
$$

Observe that $Y^{*} Y X^{2 k} \xi_{1}=\left\langle X^{2 k} \xi_{1}, \xi_{1}\right\rangle \xi_{1}=\int_{0}^{\infty} t^{k} d \sigma_{1}(t) \cdot \xi_{1}$ by (1d), while $Y Y^{*} \xi_{2}=\xi_{2}$. Therefore

$$
\left(A^{*} A\right)\left(A A^{*}\right)^{k}=\int_{0}^{\infty} t^{k} d \sigma_{1}(t) \cdot\left(\xi_{1} \otimes X^{2} \xi_{2}\right)=\int_{0}^{\infty} t^{k} d \sigma_{1}(t) \cdot A^{*} A \xi
$$

thus proving (3).

Corollary 5.7. Let $W=W_{1} W_{2} \cdots W_{d}$ be a mixed-alternating word in $A$ and $A^{*}$, factored as in (2.7) with $d \geq 2$. Then

$$
W \xi=\varphi_{\xi}\left(W_{2}\right) \varphi_{\xi}\left(W_{3}\right) \cdots \varphi_{\xi}\left(W_{d}\right) W_{1} \xi .
$$

Proof. Parts (3) and (6) of the preceding lemma yield the conclusion when $d=2$. The general case follows easily by induction on $d$.

Proposition 5.8. Let $\sigma_{1}$ and $\sigma_{2}$ be probability measures in $\mathcal{P}_{c}^{+}$, and let the operator $A$ in $\left(\mathcal{B}(\mathcal{K}), \varphi_{\xi}\right)$ be constructed as in Remark 5.3. Then the $*$-distribution of $A$ is $\eta$-diagonal. Moreover, the distributions of $A A^{*}$ and $A^{*} A$ are $\sigma_{1}$ and $\sigma_{2}$, respectively.

Proof. The second assertion follows from parts (1) and (4) of Lemma 5.5. It remains to prove that the distribution of $A$ is $\eta$-diagonal, and to do this we verify the conditions in Theorem 2.8. Let $W=W_{1} W_{2} \cdots W_{d}$ be a mixed-alternating word in $A$ and $A^{*}$, factored as in (2.7). Corollary 5.7 yields

$$
\varphi_{\xi}(W)=\varphi_{\xi}\left(W_{2}\right) \varphi_{\xi}\left(W_{3}\right) \cdots \varphi_{\xi}\left(W_{d}\right)\left\langle W_{1} \xi, \xi\right\rangle=\prod_{j=1}^{d} \varphi_{\xi}\left(W_{j}\right),
$$


thus verifying condition ( $\eta$ DM2). Finally we verify condition ( $\eta$ DM1). Suppose that $V$ is a word in $A$ and $A^{*}$ that is not mixed-alternating, and choose a mixed-alternating word $W$ of maximum length with the property that $V$ can be written as $V=U W$ for some non-empty word $U$. Also, write $W=W_{1} W_{2} \cdots W_{d}$ as in (2.7). We have

$$
\varphi_{\xi}(V)=\langle V \xi, \xi\rangle=\prod_{j=2}^{d} \varphi_{\xi}\left(W_{j}\right)\left\langle U W_{1} \xi, \xi\right\rangle
$$

by Corollary 5.7. If $|U|=1$, the equality $\varphi_{\xi}(V)=0$ follows from Lemma 5.5. If $|U| \geq 2$, then $U$ is of the form $U^{\prime} A A$ or $U^{\prime} A^{*} A^{*}$ for some (possibly empty) word $U^{\prime}$. In this case, $\varphi_{\xi}(V)=0$ by Lemma 5.6.

We conclude the discussion of the parametrization announced at the beginning of the section.

Proof of Theorem 5.2. We first note that the map $\Phi$ is well-defined. Indeed, let $\sigma_{1}, \sigma_{2} \in \mathcal{P}_{c}^{+}$be given. The existence of an $\eta$-diagonal $*$-distribution $\mu \in \mathcal{D}_{c}(1, *)$ which fulfils the conditions (5.2) is ensured by Proposition 5.8. The uniqueness of $\mu$ follows from the fact that an $\eta$-diagonal $*$-distribution is completely determined by its alternating *-moments, as we saw in Theorem 2.8 .

The surjectivity of $\Phi$ is immediate from its definition: every $\eta$-diagonal $*$-distribution $\mu \in \mathcal{D}_{c}(1, *)$ can be written as $\Phi\left(\sigma_{1}, \sigma_{2}\right)$, where $\sigma_{1}, \sigma_{2} \in \mathcal{P}_{c}^{+}$are the distributions of $Z Z^{*}$ and respectively $Z^{*} Z$ in $\left(\mathbb{C}\left\langle Z, Z^{*}\right\rangle, \mu\right)$, in the sense discussed in Definition 5.1.

Finally, the injectivity of $\Phi$ is immediate as well. Indeed, if $\Phi\left(\sigma_{1}, \sigma_{2}\right)=\mu$, then the moments of $\sigma_{1}$ and $\sigma_{2}$ can be retrieved as alternating moments of $\mu$, and compactly supported probability measures on $\mathbb{R}$ are determined by their moments.

\section{Parametrization of infinitely divisble $R$-diagonal distributions}

In this section we use the BBP method to characterize $\boxplus$-infinitely divisible $R$-diagonal distributions. The parametrization mentioned in the title of the section arises naturally, in the way indicated in the following remark.

Remark and Notation 6.1. Let $\mathcal{R}_{c}^{\text {(inf-div) }}$ denote the set of all the $R$-diagonal distributions in $\mathcal{D}_{c}(1, *)$ that are $\boxplus$ infinitely divisible. It is immediate that the bijection $\mathbb{B}_{(1, *)}$ from Theorem 4.2 induces a bijection (still denoted $\mathbb{B}_{(1, *)}$ )

$$
\mathbb{B}_{(1, *)}:\left\{\mu \in \mathcal{D}_{c}(1, *): \mu \text { is } \eta \text {-diagonal }\right\} \rightarrow \mathcal{R}_{c}^{\text {(inf-div) }} .
$$

On the other hand, Theorem 5.2 provides a natural bijection

$$
\Phi: \mathcal{P}_{c}^{+} \times \mathcal{P}_{c}^{+} \rightarrow\left\{\mu \in \mathcal{D}_{c}(1, *): \mu \text { is } \eta \text {-diagonal }\right\}
$$

The map

$$
\Psi:=\mathbb{B}_{(1, *)} \circ \Phi: \mathcal{P}_{c}^{+} \times \mathcal{P}_{c}^{+} \rightarrow \mathcal{R}_{c}^{\text {(inf-div) }}
$$

is therefore a bijection as well. We refer to $\Psi$ as the $B B P$ parametrization of $\mathcal{R}_{c}^{\text {(inf-div) }}$. Every choice of parameters $\sigma_{1}, \sigma_{2} \in \mathcal{P}_{c}^{+}$yields a distribution $v=\Psi\left(\sigma_{1}, \sigma_{2}\right) \in \mathcal{R}_{c}^{\text {(inf-div) }}$, and every $v \in \mathcal{R}_{c}^{\text {(inf-div) }}$ arises from a unique pair $\sigma_{1}, \sigma_{2}$.

We emphasize that the bijection $\Psi$ works in a really straightforward way: the coefficients of the $\eta$-series of $\sigma_{1}$ and $\sigma_{2}$ give the determining sequences of $v=\Psi\left(\sigma_{1}, \sigma_{2}\right)$. It is actually worth recording a direct consequence of this fact, as follows.

Notation 6.2. We denote by $\mathcal{E}_{c}^{+}$the collection of those series $f \in \mathbb{C} \llbracket z \rrbracket$ with the property that $f=\eta_{\sigma}$ for some $\sigma \in \mathcal{P}_{c}^{+}$(where $\sigma$ is, a fortiori, uniquely determined).

Proposition 6.3. Let $v \in \mathcal{D}_{c}(1, *)$ be an $R$-diagonal distribution, and let $\left(\alpha_{n}\right)_{n=1}^{\infty}$ and $\left(\beta_{n}\right)_{n=1}^{\infty}$ be its determining sequences. Set $f(z)=\sum_{n=1}^{\infty} \alpha_{n} z^{n}$ and $g(z)=\sum_{n=1}^{\infty} \beta_{n} z^{n}$. Then $v$ is $\boxplus$-infinitely divisible if and only if both $f$ and $g$ belong to $\mathcal{E}_{c}^{+}$. 
Proof. If $v \in \mathcal{R}_{c}^{\text {(inf-div) }}$, then $v=\Psi\left(\sigma_{1}, \sigma_{2}\right)$ for some $\sigma_{1}, \sigma_{2} \in \mathcal{P}_{c}^{+}$, hence $f=\eta_{\sigma_{1}}$ and $g=\eta_{\sigma_{2}}$, and so $f, g \in \mathcal{E}_{c}^{+}$. Conversely, suppose that $f, g \in \mathcal{E}_{c}^{+}$, so $f=\eta_{\sigma_{1}}$ and $g=\eta_{\sigma_{2}}$ for some $\sigma_{1}, \sigma_{2} \in \mathcal{P}_{c}^{+}$. Then the distribution $\widetilde{v}:=\Psi\left(\sigma_{1}, \sigma_{2}\right)$ belongs to $\mathcal{R}_{c}^{\text {(inf-div) }}$, and the definition of $\Psi$ shows that $\widetilde{v}$ has the same determining sequences as $v$. This forces $v=\widetilde{v}$, hence $v \in \mathcal{R}_{c}^{\text {(inf-div) }}$.

The criterion provided by Proposition 6.3 is useful because one can (following the work in [2]) characterize the series from $\mathcal{E}_{c}^{+}$in terms of the associated analytic functions. We will follow up on this in the application presented in Section 7.

Since we are dealing with free probabilistic structures, it is natural to ask what is the description of the BBP parametrization $\Psi$ in terms of $R$-transforms. Recall (Remark 3.4) that an $R$-diagonal $*$-distribution $v \in \mathcal{D}_{c}(1, *)$ is uniquely determined by the $R$-transforms $R_{Z Z^{*}}, R_{Z^{*} Z} \in \mathbb{C} \llbracket z \rrbracket$. The following result thus provides an alternative characterization of $\Psi\left(\sigma_{1}, \sigma_{2}\right)$.

Theorem 6.4. Let $\sigma_{1}, \sigma_{2} \in \mathcal{P}_{c}^{+}$and set $v=\Psi\left(\sigma_{1}, \sigma_{2}\right)$. Then the $R$-transforms of $Z Z^{*}$ and of $Z * Z$ in the $*$-probability space $\left(\mathbb{C}\left\langle Z, Z^{*}\right\rangle, v\right)$ are described as follows:

$$
R_{Z Z^{*}}(z)=R_{\mathbb{B}\left(\sigma_{1}\right)}\left(z\left(1+M_{\mathbb{B}\left(\sigma_{2}\right)}(z)\right)\right), \quad R_{Z^{*} Z}(z)=R_{\mathbb{B}\left(\sigma_{2}\right)}\left(z\left(1+M_{\mathbb{B}\left(\sigma_{1}\right)}(z)\right)\right),
$$

where $\mathbb{B}\left(\sigma_{1}\right)$ and $\mathbb{B}\left(\sigma_{2}\right)$ indicate the original BBP bijection (as discussed in Remark 4.5 ).

Proof. We set $\mu:=\Phi\left(\sigma_{1}, \sigma_{2}\right)$, so $v$ is $R$-diagonal, $\mu$ is $\eta$-diagonal, and $\mathbb{B}_{(1, *)}(\mu)=v$. Thus

$$
R_{\nu}\left(z, z^{*}\right)=\eta_{\mu}\left(z, z^{*}\right)=\sum_{n=1}^{\infty} \alpha_{n}\left(z z^{*}\right)^{n}+\sum_{n=1}^{\infty} \beta_{n}\left(z^{*} z\right)^{n},
$$

where $\left(\alpha_{n}\right)_{n=1}^{\infty}$ and $\left(\beta_{n}\right)_{n=1}^{\infty}$ are the (common) determining sequences for $\mu$ and for $\nu$. By the definition of the bijection $\Phi$ in Theorem 5.2, $\sigma_{1}$ has the same moments as the element $Z Z^{*}$ in the noncommutative probability space $\left(\mathbb{C}\left\langle Z, Z^{*}\right\rangle, \mu\right)$. This implies that $\eta_{\sigma_{1}}=\eta_{Z Z^{*}}$, and then Proposition 2.13 gives us the formula $\eta_{\sigma_{1}}(z)=\sum_{n=1}^{\infty} \alpha_{n} z^{n}$. In a similar way we find that $\eta_{\sigma_{2}}(z)=\sum_{n=1}^{\infty} \beta_{n} z^{n}$.

Consider now the probability measures $\mathbb{B}\left(\sigma_{1}\right), \mathbb{B}\left(\sigma_{2}\right) \in \mathcal{P}_{c}$. The definition of $\mathbb{B}$ implies

$$
R_{\mathbb{B}\left(\sigma_{1}\right)}(z)=\eta_{\sigma_{1}}(z)=\sum_{n=1}^{\infty} \alpha_{n} z^{n}, \quad R_{\mathbb{B}\left(\sigma_{2}\right)}(z)=\eta_{\sigma_{2}}(z)=\sum_{n=1}^{\infty} \beta_{n} z^{n} .
$$

But then Proposition 3.5 applies to the $R$-diagonal $*$-distribution $v$ and yields (6.4).

As a consequence of Theorem 6.4, we obtain a natural connection between the notions of $\boxplus$-infinite divisibility in $\mathcal{D}_{c}(1, *)$ and in $\mathcal{P}_{c}$. This is stated in the next corollary. The converse of the corollary fails even in the tracial framework (see Remark 6.9 below).

Corollary 6.5. Let $v \in \mathcal{D}_{c}(1, *)$ be $R$-diagonal and let $\tau_{1}, \tau_{2} \in \mathcal{P}_{c}^{+}$be the distributions of $Z Z^{*}$ and $Z^{*} Z$ in the *probability space $\left(\mathbb{C}\left\langle Z, Z^{*}\right\rangle, v\right)$ (as discussed in Definition 5.1). If $v$ is $\boxplus$-infinitely divisible in $\mathcal{D}_{c}(1, *)$, then $\tau_{1}$ and $\tau_{2}$ are $\boxplus$-infinitely divisible in $\mathcal{P}_{c}$.

Proof. By symmetry, it suffices to show that $\tau_{1}$ is $\boxplus$-infinitely divisible. According to [19, Theorem 4.3], a compactly supported Borel probability measure on $\mathbb{R}$ is $\boxplus$-infinitely divisible if and only if its $R$-transform can be extended to an analytic self-map of the upper half-plane $\mathbb{C}^{+}$. Suppose that $v=\Phi\left(\sigma_{1}, \sigma_{2}\right)$, where $\sigma_{1}, \sigma_{2} \in \mathcal{P}_{c}^{+}$. The $R$-transform $R_{\tau_{1}}$, which is the same as $R_{Z Z^{*}}$, is given by the first equation (6.4). By [6, Proposition 6.1], the moment series of the probability measure $\mathbb{B}\left(\sigma_{2}\right)$ can be extended analytically to $\mathbb{C}^{+}$and this extension satisfies

$$
z \in \mathbb{C}^{+} \Rightarrow z\left(1+M_{\mathbb{B}\left(\sigma_{2}\right)}(z)\right) \in \mathbb{C}^{+} .
$$


Finally, since $\mathbb{B}\left(\sigma_{1}\right)$ is $\boxplus$-infinitely divisible, [19, Theorem 4.3] assures us that $R_{\mathbb{B}\left(\sigma_{1}\right)}$ extends analytically to a selfmap of $\mathbb{C}^{+}$. We conclude that for every $z \in \mathbb{C}^{+}, R_{\mathbb{B}\left(\sigma_{1}\right)}$ is defined at $z\left(1+M_{\mathbb{B}\left(\sigma_{2}\right)}(z)\right)$, and that

$$
z \mapsto R_{\mathbb{B}\left(\sigma_{1}\right)}\left(z\left(1+M_{\mathbb{B}\left(\sigma_{2}\right)}\right)(z)\right)
$$

is an analytic self-map on $\mathbb{C}^{+}$, as required.

In the remainder of this section, we discuss the KMS example. In this special case one can process further the formulas from Theorem 6.4 and arrive at explicit formulas (stated in Proposition 6.8) for the distributions of $Z Z^{*}$ and of $Z^{*} Z$ in terms of the probability measures $\sigma_{1}, \sigma_{2}$ that parametrize $v$. These formulas call on some commonly used operations from the free harmonic analysis of $\mathcal{P}_{c}^{+}$, that are reviewed in the following remark.

Remark 6.6 (Some elements of free harmonic analysis on $\mathcal{P}_{c}^{+}$). (1) Measures $\sigma \in \mathcal{P}_{c}$ have free additive convolution powers with real exponent $t \in[1, \infty)$. More precisely, for every $\sigma \in \mathcal{P}_{c}$ and $t \in[1, \infty)$, there exists a unique measure $\tau \in \mathcal{P}_{c}$ such that $R_{\tau}=t R_{\sigma}$ (see [15, pp. 228-231]). This measure $\tau$ is denoted $\sigma^{\boxplus t}$. When $t$ is an integer, $\sigma^{\boxplus t}$ is simply the $t$-fold convolution $\sigma \boxplus \cdots \boxplus \sigma$. The argument in [15, pp. 228-231] also shows that $\sigma^{\boxplus t} \in \mathcal{P}_{c}^{+}$for all $t \in[1, \infty)$ if $\sigma \in \mathcal{P}_{c}^{+}$.

The analogous result for Boolean convolution provides for every $\sigma \in \mathcal{P}_{c}$ and $t \in(0, \infty)$ a Boolean convolution power $\sigma^{\uplus t} \in \mathcal{P}_{c}$ such that $\eta_{\sigma^{\uplus t}}=t \eta_{\sigma}$ (see [18, Theorem 3.6]). As in the free case, $\sigma^{\uplus t} \in \mathcal{P}_{c}^{+}$for every $t \in(0, \infty)$ if $\sigma \in \mathcal{P}_{c}^{+}$(see, for instance, the operator model constructed in [3, Proposition 4.8]).

(2) The original BBP bijection $\mathbb{B}: \mathcal{P}_{c} \rightarrow \mathcal{P}_{c}^{\text {(inf-div) }}$ (Remark 4.5) can be expressed using convolution powers, by the formula

$$
\mathbb{B}(\sigma)=\left(\sigma^{\boxplus 2}\right)^{\uplus 1 / 2}, \quad \sigma \in \mathcal{P}_{c},
$$

which was proved in [4, Theorem 1.2]). The facts reviewed in (1) above imply that $\mathbb{B}(\sigma) \in \mathcal{P}_{c}^{+}$for every $\sigma \in \mathcal{P}_{c}^{+}$.

(3) Free multiplicative convolution $\otimes$ is another binary operation defined on the set $\mathcal{P}_{c}^{+}$. This operation corresponds to the product of free random variables. Quite remarkably, $\mathbb{B} \mid \mathcal{P}_{c}^{+}$was shown in [4, Remark 3.9] to be a homomorphism for $\otimes$, that is,

$$
\mathbb{B}\left(\sigma \otimes \sigma^{\prime}\right)=\mathbb{B}(\sigma) \otimes \mathbb{B}\left(\sigma^{\prime}\right), \quad \sigma, \sigma^{\prime} \in \mathcal{P}_{c}^{+} .
$$

(4) The free counterpart of the standard Poisson distribution is the Marchenko-Pastur distribution $\Pi_{1}$ (also known as the the free Poisson distribution). This distribution is supported on the interval $[0,4]$ and it is Lebesgue absolutely continuous with density

$$
d \Pi_{1}(t) / d t=\frac{1}{2 \pi} \sqrt{(4-t) / t}, \quad 0<t \leq 4 .
$$

Its $R$-transform is

$$
R_{\Pi_{1}}(z)=z /(1-z)
$$

and a simple calculation using the definition of $\mathbb{B}$ shows that

$$
\Pi_{1}=\mathbb{B}\left(\frac{1}{2}\left(\delta_{0}+\delta_{2}\right)\right)
$$

A useful property of $\Pi_{1}$ is that it converts moment series into $R$-transforms via the formula

$$
R_{\sigma \otimes \Pi_{1}}=M_{\sigma}, \quad \sigma \in \mathcal{P}_{c}^{+} .
$$

See, for instance, [15, Propositions 17.2 and 17.4]. 
Remark 6.7. Let $\sigma \in \mathcal{P}_{c}^{+}$and let $t>0$ be a real number. The determining sequences $\left(\alpha_{n}\right)_{n=1}^{\infty}$ and $\left(\beta_{n}\right)_{n=1}^{\infty}$ of the infinitely divisible $R$-diagonal $*$-distribution $v:=\Psi\left(\sigma^{\uplus t}, \sigma\right) \in \mathcal{R}_{c}^{\text {(inf-div) }}$ satisfy

$$
\sum_{n=1}^{\infty} \beta_{n} z^{n}=\eta_{\sigma}(z), \quad \sum_{n=1}^{\infty} \alpha_{n} z^{n}=\eta_{\sigma^{\uplus t}}(z)=t \eta_{\sigma}(z) .
$$

Thus $v$ satisfies the KMS condition with parameter $t: \alpha_{n}=t \beta_{n}, n \in \mathbb{N}$ (Definition 3.6).

Proposition 6.8. With the notation of the preceding remark, let $\tau_{1}, \tau_{2} \in \mathcal{P}_{c}^{+}$be the distributions of $Z Z^{*}$ and of $Z^{*} Z$ in the noncommutative probability space $\left(\mathbb{C}\left\langle Z, Z^{*}\right\rangle, v\right)$ (as discussed in Definition 5.1). Then

$$
\tau_{1}=\left(\mathbb{B}(\sigma) \otimes \Pi_{1}\right)^{\boxplus t} \quad \text { and } \quad \tau_{2}=\left(\mathbb{B}(\sigma)^{\boxplus t} \otimes \Pi_{1}\right)^{\boxplus 1 / t} .
$$

Proof. The two formulas in (6.7) have similar proofs. We only verify the first one. Since $\tau_{1}$ is the distribution of $Z Z^{*}$, we have $R_{\tau_{1}}=R_{Z Z^{*}}$, and Theorem 6.4 yields

$$
R_{\tau_{1}}(z)=R_{\mathbb{B}\left(\sigma_{1}\right)}\left(z\left(1+M_{\mathbb{B}\left(\sigma_{2}\right)}(z)\right)\right),
$$

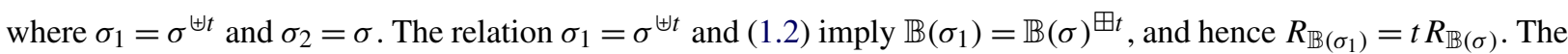
equality (6.8) can be continued as follows:

$$
\begin{aligned}
R_{\tau_{1}}(z) & =t \cdot R_{\mathbb{B}(\sigma)}\left(z\left(1+M_{\mathbb{B}(\sigma)}(z)\right)\right) \\
& =t \cdot M_{\mathbb{B}(\sigma)}(z) \quad(\text { by }(3.4)) \\
& =t \cdot R_{\mathbb{B}(\sigma) \bigotimes \Pi_{1}}(z) \quad(\text { by }(6.6)) \\
& =R_{\left(\mathbb{B}(\sigma) \otimes \Pi_{1}\right)^{\boxplus t}}(z) .
\end{aligned}
$$

Thus the probability measures $\tau_{1}$ and $\left(\mathbb{B}(\sigma) \otimes \Pi_{1}\right)^{\boxplus t}$ are equal because they have the same $R$-transform.

Remark 6.9 (Tracial case). In the special case when $t=1$, the preceding proposition reduces to

$$
\tau_{1}=\tau_{2}=\mathbb{B}(\sigma) \otimes \Pi_{1} .
$$

Using (6.5) and invoking the multiplicativity of $\mathbb{B}$ (Remark 6.6(3)), we can rewrite (6.9) as

$$
\tau_{1}=\tau_{2}=\mathbb{B}\left(\sigma \otimes \frac{1}{2}\left(\delta_{0}+\delta_{2}\right)\right) .
$$

This confirms the fact (Corollary 6.5) that $\tau_{1}$ and $\tau_{2}$ are $\boxplus$-infinitely divisible in $\mathcal{P}_{c}$.

We conclude with an argument showing that the converse of Corollary 6.5 does not hold. Choose a distribution $\tilde{\sigma} \in \mathcal{P}_{c}^{+}$that cannot be written as $\sigma \otimes \frac{1}{2}\left(\delta_{0}+\delta_{2}\right)$ for any $\sigma \in \mathcal{P}_{c}^{+}$. (For instance, $\widetilde{\sigma}=\frac{1}{3}\left(\delta_{0}+\delta_{1}+\delta_{2}\right)$ is such a distribution.) Let $v \in \mathcal{D}_{c}(1, *)$ be the tracial $R$-diagonal $*$-distribution defined by the requirement that the common distribution of $Z Z^{*}$ and $Z^{*} Z$ in $\left(\mathbb{C}\left\langle Z, Z^{*}\right\rangle, v\right)$ is equal to $\mathbb{B}(\widetilde{\sigma})$ (see [15, Proposition 15.13] for an argument that $v$ exists). The distributions of $Z Z^{*}$ and $Z^{*} Z$ are $\boxplus$-infinitely divisible in $\mathcal{P}_{c}$, by construction. We show that $v$ is not $\boxplus$-infinitely divisible in $\mathcal{D}_{c}(1, *)$. Suppose, to get a contradiction, that $v$ is $\boxplus$-infinitely divisible. Then $v=\Psi(\sigma, \sigma)$ for some $\sigma \in \mathcal{P}_{c}^{+}$. Since $\tau_{1}=\tau_{2}=\mathbb{B}(\widetilde{\sigma}),(6.10)$ yields $\mathbb{B}(\widetilde{\sigma})=\mathbb{B}\left(\sigma \otimes \frac{1}{2}\left(\delta_{0}+\delta_{2}\right)\right)$, and thus $\widetilde{\sigma}=\sigma \otimes \frac{1}{2}\left(\delta_{0}+\delta_{2}\right)$ because $\mathbb{B}$ is injective, contrary to the choice of $\widetilde{\sigma}$.

Example 6.10 ( $\lambda$-circular distribution). Let $\lambda>0$ be a parameter. If in the setting of Remark 6.7 and Proposition 6.8 we take $\sigma=\delta_{1}$ (Dirac mass at 1) and $t=\lambda$, then the resulting $*$-distribution $v \in \mathcal{R}_{c}^{\text {(inf-div) }}$ is the $\lambda$-circular distribution mentioned right before Definition 3.6. Indeed, it is immediate that in this case the series $\sum_{n=1}^{\infty} \beta_{n} z^{n}$ and $\sum_{n=1}^{\infty} \alpha_{n} z^{n}$ 
from Remark 6.7 are reduced to $\eta_{\delta_{1}}(z)=z$ and respectively to $t \eta_{\delta_{1}}(z)=\lambda z$; hence we have $\alpha_{1}=\lambda, \beta_{1}=1$ and $\alpha_{n}=\beta_{n}=0$ for all $n \geq 2$, as required in the definition of the $\lambda$-circular distribution.

In this example, the formulas indicated in Proposition 6.8 for the distributions of $Z Z^{*}$ and of $Z^{*} Z$ give free Poisson distributions. In order to make this precise, we need to review another bit of notation: for any two parameters $p, q>0$ one has a free Poisson distribution of rate $p$ and jump size $q$, which we will denote as $\Pi_{p ; q}$, and which appears in the free analogue of the Poisson limit theorem (see e.g. Proposition 12.11 in [15]). The Marchenko-Pastur distribution reviewed in Remark 6.6(4) corresponds to $p=q=1$ (so " $\Pi_{1}$ " from there becomes " $\Pi_{1 ; 1}$ "). For general $p, q>0$, the formula given in Remark 6.6(4) for the $R$-transform of $\Pi_{1}$ extends to

$$
R_{\Pi_{p ; q}}(z)=\frac{p q z}{1-q z} .
$$

Returning to the example of the $\lambda$-circular distribution, an immediate processing of the formulas (6.4) from Theorem 6.4 gives us that the $R$-transforms of $Z Z^{*}$ and of $Z^{*} Z$ in the noncommutative probability space $\left(\mathbb{C}\left\langle Z, Z^{*}\right\rangle, v\right)$ are

$$
R_{Z Z^{*}}(z)=\frac{\lambda z}{1-z}, \quad R_{Z^{*} Z}(z)=\frac{z}{1-\lambda z} .
$$

For our example, this shows that the distributions $\tau_{1}$ and $\tau_{2}$ appearing in (6.7) (Proposition 6.8) are free Poisson distributions:

$$
\tau_{1}=\Pi_{\lambda ; 1} \quad \text { and } \quad \tau_{2}=\Pi_{1 / \lambda ; \lambda}
$$

\section{Stability of $\mathcal{R}_{c}^{\text {(inf-div) }}$ under free multiplicative convolution}

Remark 7.1. In this section we consider the operation $\otimes$ on $\mathcal{D}_{c}(1, *)$, which follows the multiplication of $*$-free random variables (cf. Definition 4.1(3)). One has the remarkable fact that whenever $\mu, \mu^{\prime} \in \mathcal{D}_{c}(1, *)$ and at least one of $\mu, \mu^{\prime}$ is $R$-diagonal, it follows that $\mu \otimes \mu^{\prime}$ is $R$-diagonal as well (see [15, Proposition 15.8]). If we make the additional assumption that both $\mu$ and $\mu^{\prime}$ are $R$-diagonal, then we have explicit formulas for the determining sequences of $\mu \otimes \mu^{\prime}$ in terms of the determining sequences of $\mu$ and of $\mu^{\prime}$. To be precise, denote the determining sequences of $\mu$ by $\left(\alpha_{n}\right)_{n=1}^{\infty},\left(\beta_{n}\right)_{n=1}^{\infty}$, and those of $\mu^{\prime}$ by $\left(\alpha_{n}^{\prime}\right)_{n=1}^{\infty},\left(\beta_{n}^{\prime}\right)_{n=1}^{\infty}$. Then the determining sequences $\left(\widehat{\alpha}_{n}\right)_{n=1}^{\infty}$, $\left(\widehat{\beta}_{n}\right)_{n=1}^{\infty}$ of $\mu \otimes \mu^{\prime}$ are given by:

$$
\left\{\begin{array}{c}
\widehat{\alpha}_{n}=\sum_{\substack{\pi \sqcup \rho \in N C(2 n) \\
\pi=\left\{V_{1}, \ldots, V_{p}\right\} \in N C(1,3, \ldots, 2 n-1) \\
\text { with } 1 \in V_{1}, \text { and } \\
\rho=\left\{W_{1}, \ldots, W_{r}\right\} \in N C(2,4, \ldots, 2 n)}} \alpha_{\left|V_{1}\right|} \beta_{\left|V_{2}\right|} \cdots \beta_{\left|V_{p}\right|} \alpha_{\left|W_{1}\right|}^{\prime} \cdots \alpha_{\left|W_{r}\right|}^{\prime}, \\
\widehat{\beta}_{n}=\sum_{\substack{\pi \sqcup \rho \in N C(2 n) \\
\pi=\left\{V_{1}, \ldots, V_{p}\right\} \in N C(1,3, \ldots, 2 n-1) \\
\text { with } 1 \in V_{1}, \text { and } \\
\rho=\left\{W_{1}, \ldots, W_{r}\right\} \in N C(2,4, \ldots, 2 n)}}^{\prime} \alpha_{\left|V_{2}\right|}^{\prime} \cdots \alpha_{\left|V_{p}\right|}^{\prime} \beta_{\left|W_{1}\right|} \cdots \beta_{\left|W_{r}\right|} .
\end{array}\right.
$$

The formulas (7.1) were proved in [11, Proposition 3.9]. They can also be rephrased in terms of equations for power series, as shown in the next proposition. The formulas (7.2) in the proposition have appeared before (but only as a conjecture, without proof), in [13, Section 5.3]. For the reader's convenience, we include the proof of how (7.2) is derived from (7.1).

Proposition 7.2. With the notation of Remark 7.1, suppose that we have elements $a, b, a^{\prime}, b^{\prime}$ in a noncommutative probability space $(\mathcal{A}, \varphi)$ such that $\{a, b\}$ is free from $\left\{a^{\prime}, b^{\prime}\right\}$ and such that

$$
R_{a}(z)=\sum_{n=1}^{\infty} \alpha_{n} z^{n}, \quad R_{b}(z)=\sum_{n=1}^{\infty} \beta_{n} z^{n}
$$




$$
R_{a^{\prime}}(z)=\sum_{n=1}^{\infty} \alpha_{n}^{\prime} z^{n}, \quad R_{b^{\prime}}(z)=\sum_{n=1}^{\infty} \beta_{n}^{\prime} z^{n}
$$

Assume moreover that $\beta_{1} \neq 0 \neq \alpha_{1}^{\prime}$, so the series $R_{b}$ and $R_{a^{\prime}}$ have inverses $R_{b}^{\langle-1\rangle}$ and $R_{a^{\prime}}^{\langle-1\rangle}$ relative to composition. Then:

$$
\left\{\begin{array}{l}
\sum_{n=1}^{\infty} \widehat{\alpha}_{n} z^{n}=\left(R_{a} \circ R_{b}^{\langle-1\rangle} \circ M_{b a^{\prime}}\right)(z), \\
\sum_{n=1}^{\infty} \widehat{\beta}_{n} z^{n}=\left(R_{b^{\prime}} \circ R_{a^{\prime}}^{\langle-1\rangle} \circ M_{a^{\prime} b}\right)(z) .
\end{array}\right.
$$

Proof. The second equation in (7.2) follows from the first one if we substitute $b^{\prime}, a^{\prime}, b$ for $a, b, a^{\prime}$, respectively. To prove the first equation, we fix an $n \in \mathbb{N}$ and we suitably structure the formula for $\widehat{\alpha}_{n}$ provided in (7.1). Let us also momentarily fix an $m \leq n$ and a set $V_{1}=\left\{2 i_{1}-1, \ldots, 2 i_{m}-1\right\}$, where $1=i_{1}<\cdots<i_{m} \leq n$. Denote $n_{k}=i_{k+1}-i_{k}$, $k=1, \ldots, m$, where $i_{m+1}=n+1$. Note that $n_{1}+\cdots+n_{m}=n$ and that $V_{1}$ can recovered from $n_{1}, \ldots, n_{m}$. Use the moment-cumulant formula as in the proof of Proposition 3.5 (using the cumulant functionals and the fact that the mixed cumulants of $a^{\prime}$ and $b$ vanish on account of freeness) to obtain

$$
\sum_{\substack{\pi \sqcup \rho \in N C(2 n) \\ \pi=\left\{V_{1}, \ldots, V_{p}\right\} \in N C(1,3, \ldots, 2 n-1) \\ \rho=\left\{W_{1}, \ldots, W_{r}\right\} \in N C(2,4, \ldots, 2 n)}} \alpha_{\left|V_{1}\right|} \beta_{\left|V_{2}\right|} \cdots \beta_{\left|V_{p}\right|} \alpha_{\left|W_{1}\right|}^{\prime} \cdots \alpha_{\left|W_{r}\right|}^{\prime}=\alpha_{m} \prod_{k=1}^{m} \varphi\left(a^{\prime}\left(b a^{\prime}\right)^{n_{k}-1}\right) .
$$

Letting $V_{1}$ vary, (7.1) yields, for the $n \in \mathbb{N}$ that we had fixed:

$$
\widehat{\alpha}_{n}=\sum_{m=1}^{n} \alpha_{m} \sum_{n_{1}+\cdots+n_{m}=n} \prod_{k=1}^{m} \varphi\left(a^{\prime}\left(b a^{\prime}\right)^{n_{k}-1}\right) .
$$

We now let $n$ vary in $\mathbb{N}$, and get that

$$
\sum_{n=1}^{\infty} \widehat{\alpha}_{n} z^{n}=\sum_{n=1}^{\infty} \alpha_{m}(g(z))^{m}=R_{a}(g(z))
$$

where

$$
g(z)=\sum_{n=1}^{\infty} \varphi\left(a^{\prime}\left(b a^{\prime}\right)^{n-1}\right) z^{n} \in \mathbb{C} \llbracket z \rrbracket .
$$

It remains to show that the series $g$ introduced in (7.4) is equal to $R_{b}^{\langle-1\rangle} \circ M_{b a^{\prime}}$ or, equivalently, that one has $R_{b} \circ g=M_{b a^{\prime}}$. To see this, apply again the moment-cumulant formula (using, as in [15, Theorem 14.4] the fact that $b$ is free from $a^{\prime}$ ) to obtain

$$
\varphi\left(\left(b a^{\prime}\right)^{n}\right)=\sum_{\substack{\pi \sqcup \rho \in N C(2 n) \\ \pi=\left\{V_{1}, \ldots, V_{p}\right\} \in N C(1,3, \ldots, 2 n-1) \\ \rho=\left\{W_{1}, \ldots, W_{r}\right\} \in N C(2,4, \ldots, 2 n)}} \beta_{\left|V_{1}\right|} \cdots \beta_{\left|V_{p}\right|} \alpha_{\left|W_{1}\right|}^{\prime} \cdots \alpha_{\left|W_{r}\right|}^{\prime} .
$$

In (7.5) we can list the blocks of $\pi$ such that $1 \in V_{1}$. A similar argument to the one used above to structure the formula for $\widehat{\alpha}_{n}$ shows now that

$$
\varphi\left(\left(b a^{\prime}\right)^{n}\right)=\sum_{m=1}^{n} \beta_{m} \sum_{n_{1}+\cdots+n_{m}=n} \prod_{k=1}^{m} \varphi\left(a^{\prime}\left(b a^{\prime}\right)^{n_{k}-1}\right), \quad n \in \mathbb{N},
$$

and this implies the desired relation $M_{b a^{\prime}}=R_{b} \circ g$. 
Remark 7.3. In the notation of Remark 7.1, consider the case (disjoint from the one covered by the preceding proposition) when $\beta_{n}=0$ for every $n \in \mathbb{N}$. In anticipation of the proof of Theorem 7.8 below, it is useful to record that in this case we have

$$
\widehat{\alpha}_{n}=\alpha_{n}\left(\alpha_{1}^{\prime}\right)^{n}, \quad \forall n \in \mathbb{N} .
$$

Indeed, in this case the only way to get a non-zero term in the sum on the right-hand side of the first equation (7.1) is by requiring that $\pi=\left\{V_{1}\right\}$ with $V_{1}=\{1,3, \ldots, 2 n-1\}$. But then the condition that $\pi \sqcup \rho$ is non-crossing forces $\rho$ to be the partition of $\{2,4, \ldots, 2 n\}$ into $n$ singleton blocks. Hence the sum giving $\widehat{\alpha}_{n}$ in (7.1) is reduced to just one term, and yields the formula displayed above.

The next corollary presents a reformulation of (7.2) which has the advantage that it introduces in discussion two power series $F$ and $\widetilde{F}$, related with the subordination results of [7].

Corollary 7.4. In the framework of Proposition 7.2, we have

$$
\left\{\begin{array}{l}
\sum_{n=1}^{\infty} \widehat{\alpha}_{n} z^{n}=R_{a}\left(F(z)\left(1+M_{b}(F(z))\right)\right), \\
\sum_{n=1}^{\infty} \widehat{\beta}_{n} z^{n}=R_{b^{\prime}}\left(\widetilde{F}(z)\left(1+M_{a^{\prime}}(\widetilde{F}(z))\right)\right),
\end{array}\right.
$$

where $F=M_{b}^{\langle-1\rangle} \circ M_{b a^{\prime}}$ and $\widetilde{F}=M_{a^{\prime}}^{\langle-1\rangle} \circ M_{a^{\prime} b}$.

Proof. By symmetry, it suffices to prove the first of the two equations. Using (7.2), we see that we must verify the identity

$$
\left(R_{b}^{\langle-1\rangle} \circ M_{b a^{\prime}}\right)(z)=F(z)\left(1+M_{b}(F(z))\right) .
$$

Recalling the assumption that $\varphi(b) \neq 0$, the functional equation $M_{b}(z)=R_{b}\left(z\left(1+M_{b}(z)\right)\right)$ can be rewritten as

$$
R_{b}^{\langle-1\rangle}(w)=(1+w) M_{b}^{\langle-1\rangle}(w)
$$

(see [15, Remark 16.18]). Substitute $M_{b a^{\prime}}$ for $w$ in (7.8) to find that

$$
\left(R_{b}^{\langle-1\rangle} \circ M_{b a^{\prime}}\right)(z)=\left(1+M_{b a^{\prime}}(z)\right) \cdot\left(M_{b}^{\langle-1\rangle} \circ M_{b a^{\prime}}\right)(z)=\left(1+M_{b a^{\prime}}(z)\right) \cdot F(z) .
$$

Finally, the definition of $F$ implies that $M_{b a^{\prime}}(z)=M_{b}(F(z))$, and using this equality in the right-hand side of the preceding equality yields (7.7).

The following lemma is an immediate consequence of the definition of $\Psi$ (Remark 6.1).

Lemma 7.5. Consider a distribution $v \in \mathcal{R}_{c}^{\text {(inf-div) }}$, and let $\left(\alpha_{n}\right)_{n=1}^{\infty}$ and $\left(\beta_{n}\right)_{n=1}^{\infty}$ be its determining sequences. There exist positive elements $a, b$ in a $C^{*}$-probability space $(\mathcal{A}, \varphi)$ such that

$$
R_{a}(z)=\sum_{n=1}^{\infty} \alpha_{n} z^{n} \quad \text { and } \quad R_{b}(z)=\sum_{n=1}^{\infty} \beta_{n} z^{n} .
$$

Moreover, the distributions of $a$ and $b$ are $\boxplus$-infinitely divisible.

Proof. Write $v=\Psi\left(\sigma_{1}, \sigma_{2}\right)$, with $\sigma_{1}, \sigma_{2} \in \mathcal{P}_{c}^{+}$. Then

$$
\sum_{n=1}^{\infty} \alpha_{n} z^{n}=\eta_{\sigma_{1}}(z)=R_{\mathbb{B}\left(\sigma_{1}\right)}(z),
$$


where $\mathbb{B}\left(\sigma_{1}\right)$ is a $\boxplus$-infinitely divisible distribution in $\mathcal{P}_{c}^{+}$(cf. Remark 6.6(2)). Thus taking $a$ to be a positive element with distribution $\mathbb{B}\left(\sigma_{1}\right)$ in some $C^{*}$-probability space will fulfill the required conditions. The argument for $b$ is similar.

In reference to the set of power series $\mathcal{E}_{c}^{+}$introduced in Notation 6.2, we record a result which follows easily from [2, Proposition 2.2].

Proposition 7.6. A series $f \in \mathbb{C} \llbracket z \rrbracket$ belongs to the set $\mathcal{E}_{c}^{+}$if and only if it satisfies the following three conditions:

(i) $f$ has real coefficients;

(ii) $f$ has positive convergence radius;

(iii) $f$ can be extended to an analytic map (still denoted $f$ ) of $\mathbb{C}^{+}$into $\overline{\mathbb{C}^{+}}$such that $f(0)=0$ and $\operatorname{Arg}(z) \leq \operatorname{Arg}(f(z))$ for $z \in \mathbb{C}^{+}$.

Corollary 7.7. Suppose that the series $f(z)=\sum_{n=1}^{\infty} \alpha_{n} z^{n} \in \mathcal{E}_{c}^{+}$is not identically zero. Then $\alpha_{1}>0$.

Proof. Let $n$ be the smallest integer such that $\alpha_{n} \neq 0$ and suppose, to get a contradiction, that either $n>1$ or $n=1$ and $\alpha_{n}<0$. Choose $\gamma \in \mathbb{C}^{+}$such that $|\gamma|=1$ and $\Im\left(\gamma^{n} \alpha_{n}\right)<0$. We have $\lim _{r \downarrow 0}\left(f(r \gamma) / r^{n}\right)=\gamma^{n} \alpha_{n}$, and therefore $\Im f(r \gamma)<0$ for sufficiently small $r$, contrary to Proposition 7.6(iii).

We are now ready for the main result of this section.

Theorem 7.8. For every $v, v^{\prime} \in \mathcal{R}_{c}^{\text {(inf-div) }}$ we have $v \otimes v^{\prime} \in \mathcal{R}_{c}^{\text {(inf-div) }}$

Proof. Let $\left(\alpha_{n}\right)_{n=1}^{\infty}$ and $\left(\beta_{n}\right)_{n=1}^{\infty}$ (respectively $\left(\alpha_{n}^{\prime}\right)_{n=1}^{\infty}$ and $\left.\left(\beta_{n}^{\prime}\right)_{n=1}^{\infty}\right)$ denote the determining sequences of $v$ (respectively, $\left.v^{\prime}\right)$. Two applications of Lemma 7.5, combined with a free product construction, allow us to construct a $C^{*}$-probability space $(\mathcal{A}, \varphi)$ and positive elements $a, b, a^{\prime}, b^{\prime} \in \mathcal{A}$ such that

(a) $R_{a}(z)=\sum_{n=1}^{\infty} \alpha_{n} z^{n}$ and $R_{b}(z)=\sum_{n=1}^{\infty} \beta_{n} z^{n}$,

(b) $R_{a^{\prime}}(z)=\sum_{n=1}^{\infty} \alpha_{n}^{\prime} z^{n}$ and $R_{b^{\prime}}(z)=\sum_{n=1}^{\infty} \beta_{n}^{\prime} z^{n}$, and

(c) $\{a, b\}$ is free from $\left\{a^{\prime}, b^{\prime}\right\}$.

We know from Remark 7.1 that $v \otimes v^{\prime}$ is an $R$-diagonal distribution in $\mathcal{D}_{c}(1, *)$. Let $\left(\widehat{\alpha}_{n}\right)_{n=1}^{\infty}$ and $\left(\widehat{\beta}_{n}\right)_{n=1}^{\infty}$ denote the determining sequences of $v \otimes v^{\prime}$, and set

$$
\widehat{f}(z):=\sum_{n=1}^{\infty} \widehat{\alpha}_{n} z^{n}, \quad \widehat{g}(z):=\sum_{n=1}^{\infty} \widehat{\beta}_{n} z^{n} .
$$

By Proposition 6.3, we have to prove that $\widehat{f}, \widehat{g} \in \mathcal{E}_{c}^{+}$. By symmetry, it suffices to show that $\widehat{f} \in \mathcal{E}_{c}^{+}$, and this is done by verifying that $\widehat{f}$ satisfies conditions (i)-(iii) of Proposition 7.6. We dispose first of the simple case in which $\beta_{1}=0$. Corollary 7.7 yields $\beta_{n}=0$ for all $n \in \mathbb{N}$, and Remark 7.3 implies that $\widehat{f}(z)=\sum_{n=1}^{\infty} \alpha_{n}\left(\alpha_{1}^{\prime} z\right)^{n}$. The desired conclusion follows because $\alpha_{1}^{\prime} \geq 0$ and the series $\sum_{n=1}^{\infty} \alpha_{n} z^{n}$ belongs to $\mathcal{E}_{c}^{+}$. Similarly, if $\alpha_{1}^{\prime}=0$, Corollary 7.7 yields $\alpha_{n}^{\prime}=0$ for all $n \in \mathbb{N}$, and then (7.1) implies that $\widehat{f}=0$.

It remains to show that $\widehat{f} \in \mathcal{E}_{c}^{+}$when $\beta_{1} \neq 0 \neq \alpha_{1}^{\prime}$. In this case, Corollary 7.4 shows that $\widehat{f}=R_{a}(F(z)(1+$ $\left.\left.M_{b}(F(z))\right)\right)$, where $F=M_{b}^{\langle-1\rangle} \circ M_{b a^{\prime}}$. In other words, $\widehat{f}$ is the composition of the three power series $R_{a}(z), z(1+$ $\left.M_{b}(z)\right)$, and $F(z)$. We know that $R_{a} \in \mathcal{E}_{c}^{+}$. It was proved in [7] that $F \in \mathcal{E}_{c}^{+}$. The series $z\left(1+M_{b}(z)\right)$ also belongs to $\mathcal{E}_{c}^{+}$by [6, Proposition 6.1]. Proposition 7.6 shows that the set $\mathcal{E}_{c}^{+}$is closed under composition. Therefore $\widehat{f} \in \mathcal{E}_{c}^{+}$, thus concluding the proof.

Corollary 7.9. Suppose that $v \in \mathcal{R}_{c}^{\text {(inf-div) }}$ is the $*$-distribution of an element a in some $C^{*}$-probability space. Then the $*$-distribution of $a^{n}$ belongs to $\mathcal{R}_{c}^{\text {(inf-div) }}$ for every $n \in \mathbb{N}$. 
Proof. This follows from Theorem 7.8 and the known fact [11, Proposition 3.11] that the distribution of $a^{n}$ is equal to $v^{\otimes n}$.

We conclude the section by looking again at the KMS example, and by describing explicitly the BBP parametrization for the powers of a $\lambda$-circular element.

Remark 7.10. (1) Suppose that $v, v^{\prime} \in \mathcal{D}_{c}(1, *)$ are $R$-diagonal and satisfy the KMS condition with parameters $t, t^{\prime} \in(0, \infty)$, respectively. Consider the $*$-distribution $v \otimes v^{\prime}$, which is $R$-diagonal as well (see Remark 7.1). We claim that $v \otimes v^{\prime}$ also satisfies the KMS condition, with parameter $t t^{\prime}$. Using the same notations for determining sequences as in Remark 7.1, this claim amounts to the fact that $\widehat{\alpha}_{n}=\left(t t^{\prime}\right) \widehat{\beta}_{n}$ for every $n \in \mathbb{N}$. In order to prove this, we replace $\alpha_{\left|V_{1}\right|}$ and $\alpha_{\left|W_{1}\right|}^{\prime}$ by $t \beta_{\left|V_{1}\right|}$ and $t \beta_{\left|W_{1}\right|}^{\prime}$, respectively, in the first formula (7.1) to obtain

$$
\widehat{\alpha}_{n}=\left(t t^{\prime}\right) \sum_{\substack{\pi \sqcup \rho \in N C(2 n) \\ \pi=\left\{V_{1}, \ldots, V_{k}\right\} \in N C(1,3, \ldots, 2 n-1) \\ \rho=\left\{W_{1}, \ldots, W_{l}\right\} \in N C(2,4, \ldots, 2 n) \\ 1 \in V_{1}, 2 \in W_{1}}} \beta_{\left|W_{1}\right|}^{\prime} \alpha_{\left|W_{2}\right|}^{\prime} \cdots \alpha_{\left|W_{l}\right|}^{\prime} \beta_{\left|V_{1}\right|} \beta_{\left|V_{2}\right|} \cdots \beta_{\left|V_{k}\right|} .
$$

To see that the last sum equals $\widehat{\beta}_{n}$, we observe that pairs $(\pi, \rho)$ as above are in a bijective correspondence with pairs $(\tilde{\pi}, \widetilde{\rho})$ such that $\widetilde{\pi} \sqcup \widetilde{\rho} \in N C(2 n)$ and $\widetilde{\pi}=\left\{\widetilde{W}_{1}, \ldots, \widetilde{W}_{l}\right\} \in N C(1,3, \ldots, 2 n-1)$ and $\widetilde{\rho}=\left\{\widetilde{V}_{1}, \ldots, \widetilde{V}_{k}\right\} \in$ $N C(2,4, \ldots, 2 n)$. Indeed, $\tilde{\rho}$ and $\tilde{\pi}$ are obtained as $\tilde{\pi} \sqcup \widetilde{\rho}=\gamma_{2 n}^{-1}(\pi \sqcup \rho)$, where we use the permutation $\gamma_{2 n}$ from the proof of Proposition 3.7. Thus, the sum above is equal to

$$
\sum_{\substack{\pi^{\prime} \sqcup \rho^{\prime} \in N C(2 n) \\ \widetilde{\pi}=\left\{\widetilde{W}_{1}, \ldots, \widetilde{W}_{l}\right\} \in N C(1,3, \ldots, 2 n-1) \\ \widetilde{\rho}=\left\{\widetilde{V}_{1}, \ldots, \widetilde{V}_{k}\right\} \in N C(2,4, \ldots, 2 n) \\ 1 \in \widetilde{W}_{1}, 2 \in \widetilde{V}_{1}}} \beta_{\left|\widetilde{W}_{1}\right|}^{\prime} \alpha_{\left|\widetilde{W}_{2}\right|}^{\prime} \cdots \alpha_{\left|\widetilde{W}_{l}\right|}^{\prime} \beta_{\left|\widetilde{V}_{1}\right|} \cdots \beta_{\left|\widetilde{V}_{k}\right|},
$$

and this equals $\widehat{\beta}_{n}$ by the second formula (7.1).

(2) Now fix a real number $\lambda>0$ and consider the $\lambda$-circular distribution $v=\Psi\left(\delta_{\lambda}, \delta_{1}\right)$, as in Example 6.10. If $a$ is an element in some $*$-probability space such that the $*$-distribution of $a$ is equal to $v$, then we will say that $a$ is a $\lambda$-circular element. Such elements do of course exist, for instance we can just take $a=Z$ in the $*$-probability space $\left(\mathbb{C}\left\langle Z, Z^{*}\right\rangle, v\right)$. If $a$ is a $\lambda$-circular element, then Theorem 7.8 and Corollary 7.9 tell us that every power $a^{k}$ has $*-$ distribution $v^{\bigotimes k} \in \mathcal{R}_{c}^{\text {(inf-div) }}$. Moreover, part (1) of the present remark assures us that $v^{\bigotimes k}$ satisfies the KMS condition with parameter $\lambda^{k}$. Hence for every $k \in \mathbb{N}$ we have a BBP parametrization of the form

$$
v^{\bigotimes k}=\Psi\left(\sigma_{k}^{\uplus \lambda^{k}}, \sigma_{k}\right)
$$

for some probability measure $\sigma_{k} \in \mathcal{P}_{c}^{+}$. For $k=1$, we know from Example 6.10 that $\sigma_{1}$ is the Dirac mass $\delta_{1}$. The next proposition gives a way of describing $\sigma_{k}$ for $k \geq 2$.

Proposition 7.11. Let $\lambda$ and $\left(\sigma_{k}\right)_{k=1}^{\infty}$ be as above, and consider on the other hand the probability measures with finite $\operatorname{support}\left(\tau_{k}\right)_{k=1}^{\infty}$ defined by

$$
\tau_{k}:=\frac{\lambda^{k}}{1+\lambda^{k}} \delta_{0}+\frac{1}{1+\lambda^{k}} \delta_{1+\lambda^{k}}, \quad k \in \mathbb{N} .
$$

Then one has

$$
\sigma_{k}=\tau_{1} \otimes \cdots \otimes \tau_{k-1}, \quad k \geq 2 .
$$


Proof. As in Remark 7.10(2), we use the notation $v$ for the $\lambda$-circular distribution. We fix a $k \in \mathbb{N}$ and invoke Proposition 7.2 in the special case in which the $*$-distributions $\mu, \mu^{\prime}$ considered there are $v^{\bigotimes k}$ and $v$, respectively. The power series

$$
\sum_{n=1}^{\infty} \beta_{n} z^{n}, \quad \sum_{n=1}^{\infty} \beta_{n}^{\prime} z^{n}, \quad \sum_{n=1}^{\infty} \widehat{\beta}_{n} z^{n}
$$

from Proposition 7.2 are equal in this case to the $\eta$-series of the probability measures $\sigma_{k}, \sigma_{1}$ and $\sigma_{k+1}$, respectively. (For instance the equality $\sum_{n=1}^{\infty} \beta_{n} z^{n}=\eta_{\sigma_{k}}(z)$ follows from the comments at the end of Remark 6.1 and the fact that $v^{\otimes k}=\Psi\left(\sigma_{k}^{\uplus \lambda}, \sigma_{k}\right)$.) Note that, since $\sigma_{1}=\delta_{1}$, for the second power series in (7.10) we actually have $\sum_{n=1}^{\infty} \beta_{n}^{\prime} z^{n}=z$.

The notation of Proposition 7.2 also include some non-commutating random variables $a, b, a^{\prime}, b^{\prime}$, where $b$ is such that

$$
R_{b}(z)=\sum_{n=1}^{\infty} \beta_{n} z^{n}=\eta_{\sigma_{k}}(z)=R_{\mathbb{B}\left(\sigma_{k}\right)}(z) .
$$

From (7.11) we infer that the distribution of $b$ is $\mathbb{B}\left(\sigma_{k}\right)$. Similar reasoning, based on the formulas $R_{b^{\prime}}(z)=z$ and $R_{a^{\prime}}(z)=\lambda z$, leads to the fact that $a^{\prime}$ and $b^{\prime}$ have distributions $\delta_{\lambda}$ and $\delta_{1}$, respectively. As a consequence, we may assume without loss of generality that $a^{\prime}=\lambda$ and $b^{\prime}=1$ in their noncommutative probability space.

We are interested in the second relation (7.2) from Proposition 7.2. Due to the very simple form of $R_{a^{\prime}}$ and $R_{b^{\prime}}$, this equation simplifies to

$$
\sum_{n=1}^{\infty} \widehat{\beta}_{n} z^{n}=\frac{1}{\lambda} M_{\lambda b}(z) .
$$

The same argument as used in (7.11) shows that the left-hand side of (7.12) is equal to $R_{\mathbb{B}\left(\sigma_{k+1}\right)}(z)$. On the right-hand side of (7.12) we perform the obvious transformation $M_{\lambda b}(z)=M_{b}(\lambda z)=M_{\mathbb{B}\left(\sigma_{k}\right)}(\lambda z)$, and this leads us to a direct connection between $\sigma_{k}$ and $\sigma_{k+1}$ :

$$
R_{\mathbb{B}\left(\sigma_{k+1}\right)}(z)=\frac{1}{\lambda} M_{\mathbb{B}\left(\sigma_{k}\right)}(\lambda z)
$$

In order to make use of (7.13), it is convenient to resort to another well-known transform of free probability, the $S$-transform. For a probability measure $\sigma \in \mathcal{P}_{c}$ with non-vanishing mean, one defines the $S$-transform of $\sigma$ as the power series

$$
S_{\sigma}(z)=\frac{1}{z} R_{\sigma}^{\langle-1\rangle}(z)=\frac{z+1}{z} M_{\sigma}^{\langle-1\rangle}(z)
$$

(see, for instance, [15, Definition 18.15 and Remark 18.16 on p. 294]). Some straightforward processing of equation (7.13) (multiply both sides by $\lambda$, take inverses under composition, and write the resulting series in terms of the suitable $S$-transforms) then leads to the formula

$$
S_{\mathbb{B}\left(\sigma_{k+1}\right)}(z)=\frac{1}{1+\lambda z} S_{\mathbb{B}\left(\sigma_{k}\right)}(\lambda z) .
$$

The formula (7.14) was obtained for a fixed (but arbitrary) $k \in \mathbb{N}$. We now let $k$ vary and use a straightforward induction argument, with base case $S_{\mathbb{B}\left(\sigma_{1}\right)}(z)=S_{\delta_{1}}(z)=1$, in order to infer that

$$
S_{\mathbb{B}\left(\sigma_{k}\right)}(z)=\prod_{j=1}^{k-1} \frac{1}{1+\lambda^{j} z}, \quad \forall k \in \mathbb{N} .
$$

It remains to make the connection to the $\tau_{k}$ indicated in the statement of the proposition. For every $j \in \mathbb{N}$, an elementary calculation shows that $\mathbb{B}\left(\tau_{j}\right)$ is the free Poisson distribution $\Pi_{1 / \lambda^{j} ; \lambda^{j}}$, where the notation " $\Pi_{p ; q}$ " is as 
in Example 6.10. Another elementary calculation shows that the $S$-transform of $\Pi_{1 / \lambda^{j} ; \lambda^{j}}$ is $1 /\left(1+\lambda^{j} z\right)$. Thus the right-hand side of (7.15) can be written as $S_{\mathbb{B}\left(\tau_{1}\right)}(z) S_{\mathbb{B}\left(\tau_{2}\right)}(z) \cdots S_{\mathbb{B}\left(\tau_{k-1}\right)}(z)$.

Now, the $S$-transform is multiplicative with respect to the operation $\otimes$ ([15, Corollary 18.17]). Since $\mathbb{B}$ is multiplicative as well (Remark 6.6(3)), the observations made in the preceding paragraph lead to the formula

$$
S_{\mathbb{B}\left(\sigma_{k}\right)}=S_{\mathbb{B}\left(\tau_{1} \otimes \ldots \otimes \tau_{k-1}\right)}, \quad k \geq 2 .
$$

The required equation (7.9) follows from here, since $\mathbb{B}$ is injective and since a compactly supported probability measure with non-vanishing mean is uniquely determined by its $S$-transform.

\section{Acknowledgement}

The authors would like to thank the anonymous referee for several insightful comments on this work, and for suggesting some interesting new problems which can be considered in its continuation.

\section{References}

[1] O. Arizmendi, T. Hasebe and N. Sakuma. On the law of free subordinators. ALEA Lat. Am. J. Probab. Math. Stat. 10 (2013) $271-291$. MR3083927

[2] S. T. Belinschi and H. Bercovici. Partially defined semigroups relative to free multiplicative convolution. Int. Math. Res. Not. IMRN 2005 (2005) 65-101. MR2128863

[3] S. T. Belinschi and A. Nica. $\eta$-Series and a Boolean Bercovici-Pata bijection for bounded $k$-tuples. Adv. Math. 217 (2008) 1-41. MR2357321

[4] S. T. Belinschi and A. Nica. On a remarkable semigroup of homomorphisms with respect to free multiplicative convolution. Indiana Univ. Math. J. 57 (2008) 1679-1713. MR2440877

[5] H. Bercovici and V. Pata. Stable laws and domains of attraction in free probability theory. Ann. of Math. (2) 149 (1999) $1023-1060$. MR1709310

[6] H. Bercovici and D. Voiculescu. Free convolution of measures with unbounded support. Indiana Univ. Math. J. 42 (1993) $733-773$. MR1254116

[7] P. Biane. Processes with free increments. Math. Z. 227 (1998) 143-174. MR1605393

[8] W. Ejsmont and F. Lehner Sample variance in free probability. Preprint, 2016. Available at arXiv:1607.06586.

[9] A. Guionnet, M. Krishnapur and O. Zeitouni. The single ring theorem. Ann. of Math. (2) 174 (2011) 1189-1217. MR2831116

[10] U. Haagerup and F. Larsen. Brown's spectral distribution measure for R-diagonal elements in finite von Neumann algebras. J. Funct. Anal. 176 (2000) 331-367. MR1784419

[11] B. Krawczyk and R. Speicher. Combinatorics of free cumulants. J. Combin. Theory Ser. A 90 (2000) 267-292. MR1757277

[12] F. Lehner. Cumulants in noncommutative probability I. Noncommutative exchangeable systems. Math. Z. 248 (2004) 67-100. MR2092722

[13] A. Nica, D. Shlyakhtenko and R. Speicher. R-diagonal elements and freeness with amalgamation. Canad. J. Math. 53 (2001) $355-381$. MR1820913

[14] A. Nica and R. Speicher. R-diagonal pairs - a common approach to Haar unitaries and circular elements. Fields Inst. Commun. 12 (1997) 149-188. MR1426839

[15] A. Nica and R. Speicher. Lectures on the Combinatorics of Free Probability. London Mathematical Society Lecture Note Series 335. Cambridge University Press, Cambridge, 2006. MR2266879

[16] D. Shlyakhtenko. Free quasi-free states. Pacific J. Math. 177 (1997) 329-368. MR1444786

[17] P. Sniady and R. Speicher. Continuous family of invariant subspaces for R-diagonal operators. Invent. Math. 146 (2001) $329-363$. MR1865398

[18] R. Speicher and R. Woroudi. Boolean convolution. Fields Inst. Commun. 12 (1997) 267-279. MR1426845

[19] D. Voiculescu. Addition of certain noncommuting random variables. J. Funct. Anal. 66 (1986) 323-346. MR0839105 